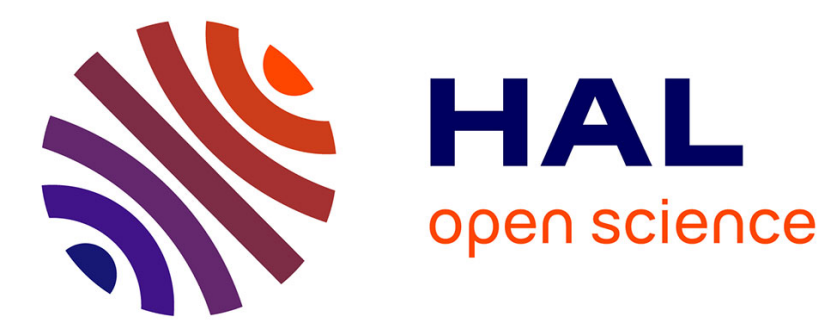

\title{
Internal gravity waves in a stratified layer atop a convecting liquid core in a non-rotating spherical shell
}

\author{
M Bouffard, B Favier, D Lecoanet, M. Le Bars
}

\section{To cite this version:}

M Bouffard, B Favier, D Lecoanet, M. Le Bars. Internal gravity waves in a stratified layer atop a convecting liquid core in a non-rotating spherical shell. Geophysical Journal International, 2022, 228 (1), pp.337-354. 10.1093/gji/ggab343 . hal-03380548

\section{HAL Id: hal-03380548 \\ https://hal.science/hal-03380548}

Submitted on 20 Oct 2021

HAL is a multi-disciplinary open access archive for the deposit and dissemination of scientific research documents, whether they are published or not. The documents may come from teaching and research institutions in France or abroad, or from public or private research centers.
L'archive ouverte pluridisciplinaire HAL, est destinée au dépôt et à la diffusion de documents scientifiques de niveau recherche, publiés ou non, émanant des établissements d'enseignement et de recherche français ou étrangers, des laboratoires publics ou privés. 


\title{
Internal gravity waves in a stratified layer atop a convecting liquid core in a non-rotating spherical shell
}

\author{
M. Bouffard ${ }^{1}$, B. Favier ${ }^{1}$, D. Lecoanet ${ }^{2,3}$ and M. Le Bars ${ }^{1}$ \\ ${ }^{1}$ CNRS, Aix Marseille Univ, Centrale Marseille, IRPHE, Marseille, France \\ ${ }^{2}$ Department of Engineering Sciences and Applied Mathematics, Northwestern University, Evanston, IL 60208, USA \\ ${ }^{3}$ CIERA, Northwestern University, Evanston, IL 60201, USA
}

43 August 2021

\section{SUMMARY}

Seismic and magnetic observations have suggested the presence of a stably stratified layer atop Earth's core. Such a layer could affect the morphology of the geomagnetic field and the evolution of the core, but the precise impact of this layer depends largely on its internal dynamics. Among other physical phenomena, stratified layers host internal gravity waves, which can be excited by adjacent convective motions. Internal waves are known to play an important role on the large scale dynamics of the Earth's climate and on the long-term evolution of stars. Yet, they have received relatively little attention in the Earth's outer core so far and deserve detailed investigations in this context. Here, we make a first step in that direction by running numerical simulations of internal gravity waves in a non-rotating spherical shell in which a stratified layer lies on top of a convective region. We use a non-linear equation of state to produce self-consistently such a two-layer system. Both propagating waves and global modes coexist in the stratified layer. We characterise the spectral properties of these waves and find that energy is distributed across a wide range of frequencies and length scales, that depends on the Prandtl number. For the control parameters considered and in the absence of rotational and magnetic effects, the mean kinetic energy in the layer is about $0.1 \%$ that of the convec- 

tive region. Gravity waves produce perturbations in the gravity field that may fall within the sensitivity limit of present-day instruments and could potentially be detected in available data. We finally provide a road map for future, more geophysically realistic, studies towards a more thorough understanding of the dynamics and impact of internal waves in a stratified layer atop Earth's core.

Key words: core dynamics- stratified layer - internal waves - numerical simulations.

\section{INTRODUCTION}

Numerous studies support the existence of a stably stratified layer atop Earth's core. Observationbased evidence for a stratification comes mostly from anomalous travel times of SmKS body waves sampling the top of the core (Helffrich \& Kaneshima, 2010; Kaneshima \& Helffrich, 2013; Kaneshima \& Matsuzawa, 2015; Tang et al., 2015; Kaneshima, 2018) and from interpretations of temporal fluctuations in the geomagnetic field (Buffett, 2014; Buffett et al., 2016). Several scenarios have been proposed for the formation of this layer. The outermost core may be thermally stratified if the core mantle boundary (CMB) heat flow is subisentropic (Labrosse, 2015). Alternatively, barodiffusion (Gubbins \& Davies, 2013), accumulation of light materials emitted by inner core crystallization (Moffatt \& Loper, 1994; Bouffard et al., 2019), chemical interactions with the mantle (Buffett \& Seagle, 2010) or immiscibility between liquid Fe-Si and Fe-Si-O in core conditions (Arveson et al., 2019) may form a stratified pool of light elements under the CMB over long time scales. On the other hand, geochemical models of core formation (Rubie et al., 2011; Jacobson et al., 2017), laboratory experiments of giant impacts (Landeau et al., 2016) and numerical simulations of convective erosion of a chemically stratified layer (Bouffard et al., 2020) all suggest that the layer may be an ancient chemical feature, formed at the same time as the core and preserved up to the present day. The properties of the layer (thickness and stratification) inferred from these various observations and models are sometimes contradictory and range from a thick (up to $450 \mathrm{~km}$ ) and strongly-stratified layer to a rather thin (100 km or less) and weakly-stratified layer. A stratified layer atop Earth's core may affect the evolution of the core (Labrosse, 2015) and the structure of the magnetic field generated by the geodynamo underneath (Christensen \& Wicht, 

2008; Nakagawa, 2011; Olson, Landeau \& Reynolds, 2017; Gastine et al., 2020). However, the

precise geophysical impacts of such a layer strongly depend on its exact structure, properties and internal dynamics, which remain largely unknown.

Even though stratification inhibits radial motions, stratified layers can have rich and complex dynamics. Several instabilities can develop in stratified environments under certain conditions, such as double diffusive convection when buoyancy is controlled by two physical fields with different molecular diffusivities (e.g. temperature and salt concentration, see for instance Schmitt, 1994 and Monville et al., 2019). Stratified layers can also host internal gravity waves (GW), which are the focus of the present study. GW are a type of waves that develops in stratified environments and for which gravity acts as a restoring force. They can be excited by an adjacent convective region (Couston et al., 2018a) or by large-scale tidal flows (Ogilvie \& Lin, 2004), and propagate in the stratified layer, transporting energy and momentum. In oceanic, atmospheric and astrophysical sciences, it is well known that GW can profoundly affect the large-scale dynamics in various ways. In the Earth's tropical stratosphere, GW drive a mean eastward/westward flow that reverses approximately every 28 months. This phenomenon, called the "quasi-biennial oscillation" (QBO), affects the entire climate system by modulating atmospheric circulation, gas distributions and seasonal weather patterns up to the poles (Baldwin et al., 2001). Similar internal wave-driven mean flows may contribute to the long-term angular momentum evolution of stars (Rogers et al., 2013; Fuller et al., 2014). Angular momentum transport by GW also enhances mixing of chemicals, which is often thought to be responsible for anomalous abundances of lithium and other elements at the surface of stars (Charbonnel \& Talon, 2005). GW are also used in asteroseismology to probe the otherwise inaccessible cores of stars (Aerts, Christensen-Dalsgaard \& Kurtz, 2010).

GW have received a marked attention in stars. Several authors performed numerical simulations of GW in the anelastic approximation or with fully compressible equations, either in a 2D equatorial plane (Rogers, Lin \& Lau, 2012; Rogers et al., 2013; Horst et al., 2020) or in a full 3D rotating sphere (Alvan, Brun \& Mathis, 2014; Edelmann et al., 2019). In the Earth's core, the properties of GW have been calculated theoretically for a fully stratified outer core (Crossley \& Rochester, 1978; Dintrans et al., 1999; Olson, 1977; Friedlander, 1987). The same authors dis- 

to detect such signals have remained unsuccessful so far. This may be because the important role played by the Lorentz and Coriolis forces in the Earth's core brings further complexity by modulating GW and allowing for other types of waves to coexist (Braginsky, 1999). For instance, Buffett (2014), Buffett et al. (2016) and Jaupart \& Buffett (2017) show that MAC waves-that arise from the interplay between Magnetic, Archimedean and Coriolis forces-could in theory explain both a 60 year fluctuation in the geomagnetic dipole and a time-dependent zonal flow at the top of the core inferred from geomagnetic secular variation. Vidal \& Schaeffer (2015) evaluated the geomagnetic signatures of a stratified layer by looking at its effect on quasi-geostrophic inertial modes in the core. However, these theoretical predictions for the properties and signatures of GW, MAC waves and inertial modes have not yet been confirmed by 3D numerical simulations. As for the dynamical effects of GW in the Earth's core, several questions remain unexplored. Notably, whether large-scale mean flows analogous to the Earth's QBO are taking place in the Earth's core is unknown. The angular momentum transport by GW in stars (Rogers et al., 2013) also suggests that $\mathrm{GW}$ in the core could deposit angular momentum on the $\mathrm{CMB}$, which would affect the length of day. Detecting unequivocal signatures of GW in the geomagnetic field, gravity data, length of day or other observables would strengthen confidence in the existence of a stratified layer below the CMB and offer another window on the dynamics of the Earth's outer core by remotely probing the local, physical properties.

Previous numerical simulations of GW excited in a self-consistent, two-layer system in nonrotating and non-magnetic Cartesian geometries by Couston et al. (2017, 2018a, 2019) showed that the waves dynamics are already very rich in simple geometries. There is thus a long way ahead from these Cartesian physical studies towards geophysical applications to planetary cores in which rotation and magnetic fields play a dominant role. This paper constitutes a first step in that direction by extending the Couston studies to a non-rotating, non-magnetic, spherical shell, the top half of which is stably stratified and lies on top of a turbulent convective region (figure 11). While a layer that occupies half of the shell is certainly much larger than expected for the core, a thick stratified layer is expected for Mercury (Christensen, 2006). In addition, a larger stratified 
layer allows us to have well-developed $\mathrm{GW}$ in the layer for the control parameters accessible in the numerical simulations. We perform a first study of the non-rotating, non-magnetic case and provide tools and concepts for characterizing the properties of GW. These will be applicable to the rotating and magnetic cases in future studies. Therefore, this study should be considered as a proof of concept providing first interesting physical insights rather than a realistic study including all geophysically relevant ingredients. In this first step, neglecting rotation and magnetic effects allows us to reach turbulent regimes at a more moderate numerical expense and to conduct a systematic study by varying parameters. We also note that while rotational effects are dominant in most planetary cores, they may be of moderate or even negligible importance in some cases, including the Earth's primitive magma ocean (Maas \& Hansen, 2019) and the outer cores of Mercury and Venus (Evonuk \& Samuel, 2012). In all these contexts, stratified layers may be present (Ohtani, 1985; Christensen et al., 2006; Jacobson et al., 2017; Laneuville et al., 2018) and would thus be in contact with a convective region only marginally influenced by rotation. The results for a non-rotating sphere derived in the present paper may apply to such cases.

The paper is divided as follows. Section 2 presents the mathematical modeling and its numerical implementation. Section 3 provides a qualitative description of the excitation and morphology of waves in the stratified layer. The spectral properties of the waves are then analysed more quantitatively in section 4 . In section 5, we quantify the energy transfer from the convective region to the waves, which allows us to evaluate potential geophysical signatures of the waves in section 6 . The final section 7 summarizes our findings and provides a road map for future studies.

\section{MATHEMATICAL AND NUMERICAL MODELING}

\subsection{Main equations}

We consider the dynamics of an incompressible fluid in a 3D spherical shell with inner and outer radii $r_{i}$ and $r_{o}$. In addition to the equation for mass conservation,

$$
\boldsymbol{\nabla} \cdot \mathbf{u}=0
$$


we solve the Navier-Stokes equation for a Newtonian non-rotating fluid in the Boussinesq approximation,

$$
\frac{\partial \mathbf{u}}{\partial t}+(\mathbf{u} \cdot \boldsymbol{\nabla}) \mathbf{u}=-\frac{1}{\rho_{0}} \boldsymbol{\nabla} \Pi+\nu \boldsymbol{\Delta} \mathbf{u}-g(r) \frac{\delta \rho}{\rho_{0}} \mathbf{e}_{\mathbf{r}}-\frac{1}{\tau(r)} \mathbf{u},
$$

where $t, \mathbf{u}$ and $\Pi$ are the time, velocity and dynamic pressure, respectively, $\rho_{0}$ a reference density and $\delta \rho$ the density perturbation which depends only on the temperature. $\nu$ is the constant kinematic viscosity, $g$ the gravity is a linear function of the radius $r$,

$$
g(r)=g_{o} \frac{r}{r_{o}}
$$

and $-\mathbf{u} / \tau(r)$ is a damping term significant only close to the top boundary, used to prevent waves reflections in one case, with $\tau$ a fixed characteristic time. The energy conservation equation is

$$
\frac{\partial T}{\partial t}+\mathbf{u} \cdot \nabla T=\kappa \nabla^{2} T
$$

where $T$ denotes the temperature and $\kappa$ the constant thermal diffusivity.

To model a stratified layer at the top of the shell in contact with a convecting region underneath, two strategies are possible. Most studies so far have imposed the stratified layer through a background temperature gradient that is positive (convectively unstable) in the bottom part and negative (stable to convection) in the top part (Christensen, 2006; Stanley and Mohammadi, 2008; Manglik, Wicht \& Christensen, 2010; Nakagawa, 2015; Christensen, 2018; Olson, Landeau \& Reynolds, 2018; Mound et al., 2019). Instead, we assume a non-linear equation of state for the density anomaly $\delta \rho$,

$$
\delta \rho / \rho_{0}=-\alpha(T)\left(T-T_{\text {inv }}\right)
$$

in which $\alpha$, the thermal expansivity, is a piecewise function of temperature,

$$
\alpha(T)=\left\{\begin{array}{rll}
\alpha_{0} & \text { if } & T \geq T_{\mathrm{inv}} \\
-S \alpha_{0} & \text { if } & T<T_{\mathrm{inv}},
\end{array}\right.
$$

with $\alpha_{0}$ the reference thermal expansivity and $S>0$ the stiffness. $T_{\mathrm{inv}}$ is an inversion temperature around which $\alpha$ changes sign. This formulation is inspired by the behavior of water around $4{ }^{\circ} \mathrm{C}$ (Townsend, 1964; Le Bars et al., 2015; Léard et al., 2020). Although such behaviour is unlikely in the Earth's outer core, this formulation allows for the formation of a self-consistent two-layer 

convective/stratified system. When a negative temperature gradient $(\partial T / \partial r<0)$ is imposed in the shell by prescribing $T=T_{i}>T_{\mathrm{inv}}$ at the inner boundary and $T=T_{o}<T_{\mathrm{inv}}$ at the outer boundary, the change of sign around $T_{\mathrm{inv}}$ in (6) divides the shell into two distinct regions (figure 1). For $T_{i} \geq$ $T \geq T_{\text {inv }}$, the negative temperature gradient is convectively unstable: the bottom part of the shell convects. For $T_{\mathrm{inv}}>T \geq T_{o}$, the temperature gradient becomes stable to convection: the top of the core is stably stratified. The interface region between the convective and stratified regions is located at the radius $r_{\text {int }}$ where $T \approx T_{\text {inv }}$. The respective sizes of the convective and stratified regions adjust so that the system reaches thermal equilibrium: at the radial position corresponding to $T=$ $T_{\text {inv }}$, the heat flux transported by convection matches the conductive heat flux in the stratified layer providing the heat flux transported by waves is negligible compared to this conductive flux (this is indeed always the case here). Another advantage of this formulation is that it is relatively straightforward to implement in pre-existing codes as only the buoyancy term is modified. We have checked that the discontinuity in $\alpha(T)$ around $T=0$ does not generate any spurious behavior in the simulation, and that the flow computed using a smooth alpha profile (using e.g. a tanh function) rapidly converges towards the flow computed using the discontinuous $\alpha(T)$ profile (6), which is hence used here. For more details on this approach, see Couston et al. (2017) and Le Bars et al. (2020). Note that our model departs from the classical Boussinesq approximation since the thermal expansion coefficient varies with temperature.

We scale distances and times using the shell thickness $D=r_{o}-r_{i}$ and the thermal diffusion time $D^{2} / \kappa$, respectively. The velocity is scaled by $\kappa / D$ and the pressure by $\left(\rho_{0} \kappa^{2} / D^{2}\right)$. The temperature scale is the temperature difference $\Delta T=T_{i}-T_{\text {inv }}$ between the bottom of the shell and the interface with the stratified region. Therefore, in non-dimensional units we set $T_{i}=1$ and $T_{\text {inv }}=0$. The dimensionless expression of equation (2) is

$$
\frac{\partial \mathbf{u}}{\partial t}+(\mathbf{u} \cdot \boldsymbol{\nabla}) \mathbf{u}=-\boldsymbol{\nabla} \Pi+\operatorname{Pr} \boldsymbol{\Delta} \mathbf{u}+\operatorname{Pr} R a T \frac{\mathbf{r}}{r_{o}}-\frac{1}{\tau(r)} \mathbf{u}
$$

where

$$
\operatorname{Pr}=\frac{\nu}{\kappa}
$$


Table 1. Parameters for the simulations. $N_{r}$ is the number of radial grid points. $\ell_{\max }$ is the maximum spherical harmonic degree. The maximal azimuthal wavenumber is $m_{\max }=\ell_{\max } \cdot \operatorname{Pr}$ is the Prandtl number, $R a_{0}$ the Rayleigh number as defined in (10), $S$ the stiffness and $T_{o}$ the imposed top temperature. $f_{N}=\bar{N} / 2 \pi$ is the mean buoyancy frequency in the stratified layer and $f_{c}=U_{\text {conv }} /(2 \pi H)$ the convective frequency. $R e_{\text {conv }}=U_{\text {conv }} H / \nu$ is the Reynolds number in the convective region that has a height $H \approx D / 2 . U_{\text {conv }}$ is the rms velocity in the convective region.

\begin{tabular}{ccccccccc} 
Simulation & $\left(N_{r}, \ell_{\max }\right)$ & $P r$ & $R a_{0}$ & $S$ & $T_{o}$ & $f_{N} / f_{c}$ & damping & $R e_{\text {conv }}$ \\
\hline P03 & $(400,160)$ & 0.3 & $4.7 \times 10^{8}$ & 30 & -6.7 & 20.1 & no & $2.518 \times 10^{3}$ \\
P1 & $(400,160)$ & 1 & $6 \times 10^{8}$ & 30 & -6.7 & 99.67 & no & $2.218 \times 10^{3}$ \\
P1_S100 & $(400,160)$ & 1 & $6 \times 10^{8}$ & 100 & -6.7 & 187.81 & no & $2.149 \times 10^{3}$ \\
P1d & $(400,160)$ & 1 & $6 \times 10^{8}$ & 30 & -6.7 & 102.94 & yes & $2.147 \times 10^{3}$ \\
P3 & $(400,160)$ & 3 & $1.2 \times 10^{9}$ & 30 & -6.7 & 457.19 & no & $2.055 \times 10^{3}$
\end{tabular}

is the Prandtl number and

$$
R a=\left\{\begin{array}{r}
R a_{0} \quad \text { if } \quad T \geq 0 \\
-\mathcal{S} R a_{0} \text { if } \quad T<0
\end{array}\right.
$$

is the Rayleigh number, with

$$
R a_{0}=\frac{\alpha_{0} g_{0} \Delta T D^{3}}{\kappa \nu} .
$$

Since the convective region extends only to a spherical shell of (dimensionless) thickness $H$, the effective Rayleigh number for the convective region is:

$$
R a_{\mathrm{eff}}=R a_{0} H^{3}
$$

Equation (4) becomes, in non-dimensional form,

$$
\frac{\partial T}{\partial t}+\mathbf{u} \cdot \nabla T=\nabla^{2} T
$$

The stratification strength in the stable region is measured by the Brunt-Väisälä frequency,

$$
N(r)=\sqrt{-\frac{g(r)}{\rho} \frac{\partial\langle\bar{\rho}\rangle}{\partial r}},
$$

where $\langle\bar{\rho}\rangle$ is the density averaged in time and azimuth. In non-dimensional form, $N$ is

$$
N(r)=\sqrt{-R a_{0} S \operatorname{Pr} \frac{\partial\langle\bar{T}\rangle}{\partial r} \frac{r}{r_{o}}},
$$


where $\langle\bar{T}\rangle$ is the time and horizontally averaged temperature and $S$ is the stiffness parameter that appears in equation (6). $S$ is therefore a measure of how "stiff" i.e. strongly stratified the stable layer is. Because both the temperature gradient and the gravity vary across the layer, $N$ varies between the interface and the top of the layer. $N$ is larger immediately above the interface and gradually decreases by about $20 \%$ towards the top of the layer. Note that this does not significantly affect the following results: for physical interpretation of the results, it is most of the time sufficient to assume the stratified layer to be linearly stratified in first approximation, with a constant Brunt-Väisälä frequency $\bar{N}$. In one simulation we introduced a damping term $-\mathbf{u} / \tau$ to prevent wave reflection at the top boundary. Note that this is not geophysically relevant to cores where rigid boundaries promote wave reflection; but as will be seen below, this will help us disentagling contributions from propagating waves and standing modes. We pick a similar form as in Couston et al. (2018a),

$$
\tau^{-1}=\pi \sqrt{R a_{0} S T_{o} / 0.5}\left[1+20 \tanh \left(r-r_{o}+0.15\right)\right]
$$

where $T_{o} / 0.5$ approximates the temperature gradient in the stratified layer of thickness $H \approx D / 2$.

We impose no-slip $(\mathbf{u}=0)$ and fixed temperature boundary conditions. The bottom boundary is set to $T=T_{i}=1$ (in non-dimensional units) while at the top boundary $T=T_{o}$. We pick the value of $T_{o}$ such that in statistically steady state, the convection zone has $H \approx D / 2$, as explained in subsection 2.2,

\subsection{Numerical method}

To solve equations (1), (7) and (12) with the non-linear equation of state (5) we use a modified version of the code PARODY (E. Dormy, J. Aubert; Dormy, 1997; Aubert et al., 2008). The equations are discretized on $N_{r}$ points in the radial direction using a classical second-order finite difference scheme on a nonuniform grid. In the two-layer system studied here, both the boundary layers and the interface region require enhanced resolution. To achieve that, we distribute 400 points along the radius so that about 240 points span the convective region, 100 points cover the interface region $\left(r_{\text {int }} \pm 0.05\right)$ and the ratio of the maximum to minimal radial spacing is 10. 
At each radius, the physical quantities are decomposed into spherical harmonics up to degree $\ell_{\max }$ and order $m_{\max }=\ell_{\max }$. To prevent aliasing errors, the numbers of grid points in the latitudinal and longitudinal directions are respectively $N_{\theta}=1.5 \ell_{\max }$ and $N_{\phi}=3 \ell_{\max }$. The non-linear terms (now including the buoyancy term) are computed in physical space on Gauss collocation points involving $N_{\theta}$ points in latitude and $N_{\phi}$ in longitude before being transformed back to spectral space. The time-integration is performed using a semi-implicit scheme, Crank-Nicolson, for diffusion and second-order Adams-Bashforth for the other terms. The waves dynamics imposes a supplementary criterion on the time step to ensure that all waves are properly resolved. As the frequency of GW is bounded by the Brunt-Väisälä frequency, we impose

$$
\Delta t=\min \left(\Delta t_{\text {conv }}, 0.1 / \bar{N}\right),
$$

where $\Delta t_{\text {conv }}$ is derived from the classic CFL criterion on the velocity. Adaptive time stepping is used to reach the statistically stationary state. Then, the time step is fixed to the minimal time step reached to avoid temporal interpolations during the computation of time spectra. Frequency spectra shown in the paper are calculated using a Hamming window method.

The top temperature $T_{o}$ for which the thickness of the convective region $H$ equals our assigned value $H=D / 2$ in thermal equilibrium is not known a priori. To obtain a first guess for $T_{o}$, we run a purely convective simulation in a "half" spherical shell comprised between radii $r_{i}$ and $r_{i}+H$ with $R a_{\text {eff }}$ as the input Rayleigh number and we measure the heat flux

$$
Q_{0}=-\frac{\mathrm{d} T}{\mathrm{~d} r}\left(r=r_{i}+H\right)
$$

at the top of this convective region. Then, when the stratified layer is added, $T_{o}$ must be such that the convective heat flux $Q_{0}$ matches the diffusive heat flux in the stratified layer above, neglecting heat flux from GW. This condition is

$$
T_{o}=\frac{\left(r_{i}+H\right)\left(r_{i}+H-r_{o}\right)}{r_{o}} Q_{0} .
$$

Following Couston et al. (2018a), we then perform iterative adjustment of $T_{o}$ to reach $H_{\text {eff }} \approx H=$ $D / 2$ at the equilibrated state. At each step of the iterative process, we run the simulation for a few 

advection times. $T_{o}$ is then adjusted such that:

$$
Q_{i}\left(\frac{r_{i}}{r_{i}+H}\right)^{2}=T_{o} \frac{r_{o}}{\left(r_{i}+H-r_{o}\right)\left(r_{i}+H\right)},
$$

where

$$
Q_{i}=-\frac{\partial T}{\partial r}\left(r_{i}\right)
$$

is the heat flux measured at the bottom of the convective region. The process repeats until $T_{o}$ varies by less than $10 \%$ between two consecutive iterations. This procedure was applied to our reference simulation at $\operatorname{Pr}=1$. Then, when changing the Prandtl number, $T_{o}$ was kept the same and only the $R a_{0}$ number was adjusted so that $H_{\text {eff }} \approx H=D / 2$ at the equilibrated state.

We explore the parameter space between $\operatorname{Pr}=0.3$ to $\operatorname{Pr}=3$. We consider large values of the stiffness $(S=30$ and $S=100$ ), which produces a strongly stratified layer and a sharp interface with the convective region (Couston et al., 2017). The Rayleigh numbers are chosen so that the convection is turbulent, characterized by Reynolds number $R e_{\text {conv }}=U_{\text {conv }} H / \nu \sim$ $2-3 \times 10^{3}$, where $U_{\text {conv }}$ and $H \approx D / 2$ are the rms velocity and the thickness of the convective region, respectively. Table 1 summarizes the parameters for all simulations. In this table we also define the buoyancy frequency $f_{N}=\bar{N} / 2 \pi$, where $\bar{N}$ is the mean Brunt-Väisälä frequency in the layer, and the convective frequency $f_{c}=U_{\text {conv }} /(2 \pi H)$, with $U_{\text {conv }}$ the rms velocity in the convective region. Note that, for the Earth's core with $\Omega=7.292 \times 10^{-5} \mathrm{~s}^{-1}, N \sim 0.7 \Omega-25 \Omega$ (for this estimation of $N$, see references given in section 6), $U_{\text {conv }} \approx 5 \times 10^{-4} \mathrm{~m} \mathrm{~s}^{-2}$ (Finlay \& Amit, 2011), we have $f_{N} / f_{c} \sim 10^{6}-10^{7} \gg 1$, i.e. the frequency of GW is large compared to the convective frequency. This is also the case in our simulations, as we have large $f_{N} / f_{c}$ ratios $\left(f_{N} / f_{c} \in[20.1-457.19]\right)$.

\section{EXCITATION OF WAVES}

\subsection{Propagating waves}

At statistically steady state, the spherical shell is divided into a convective region in the bottom and a stably stratified layer above (figure 2a). These two layers occupy one half of the shell each and are separated by a sharp interface located at $r \approx 1$ (see figure 1 for a schematic illustration 
and figure $2 \mathrm{~b}$ for a snapshot from simulation P1). Convection takes the form of a turbulent flow involving multiple length scales (figure 2p, inner half of the shell; see also movie attached). The turbulent motions that occur close to the stable layer deform slightly the interface or penetrate shortly into the layer. These interface processes couple the convective region with the stratified layer by generating internal gravity waves (GW) immediately above the interface, which then propagate in the entire stratified layer (figure $2 \mathrm{p}$, outer half of the shell; see also movie attached).

Convective plumes hit the interface and overshoot in the stratified layer (figure 3). Reynolds stresses near the conductive-convective interface transfer power from convective motions to linear waves (e.g. Lecoanet et al., 2015). GW satisfy the dispersion relation

$$
\omega=\frac{\bar{N} k_{\perp}}{\sqrt{k_{r}^{2}+k_{\perp}^{2}}},
$$

where $\omega$ is the frequency of the wave, $\mathbf{k}=k_{r} \mathbf{e}_{\mathbf{r}}+\mathbf{k}_{\perp}$ the wavevector decomposed in its radial and horizontal parts and $\bar{N}$ is the averaged Brunt-Väisälaä frequency which we assume to be constant for simplicity. Convective plumes generate waves over a large range of possible frequencies, and especially with frequency similar to the inverse convective time scale, $U_{\text {rms }} / H$, which propagate at a characteristic angle $\alpha$,

$$
\alpha=\arccos \frac{\omega}{\bar{N}} .
$$

Therefore, GW transport energy away along a cone originating from the impact point. The intersection of this cone with a spherical surface $(r=\mathrm{cst})$ is a circle. At a given radius in the stratified layer, several circular patterns can be seen in the velocity field, each one being the trace of the waves produced by a plume impact underneath (figure 4). In a plane perpendicular to the interface and containing the impact point, the waves form a characteristic shape sometimes referred to as a "half St Andrew's cross" (figure 3 middle). The group velocity $\mathbf{v}_{\mathbf{g}}$, at which the envelope of the waves and hence the energy are transported, is perpendicular to the phase velocity $\mathbf{v}_{\mathbf{p}}$ and inclined at the angle $\alpha$ from the local vertical (figure 3). Along their course, GW are attenuated owing to viscous and thermal dissipation, and due to a geometrical effect in a sphere, so that the intensity of propagative waves is maximal immediately above the impact point and decreases as the 

waves spread out (figure 5). Attenuation is larger for high wavenumbers and/or lower frequencies (Lecoanet et al., 2015).

In addition, there are also waves that are secularly generated by convective Reynolds stresses not associated with plumes. These predominantly excite lower frequency waves, and are harder to see by eye, as they are being continuously generated throughout the convection zone (Lecoanet et al., 2015).

\subsection{Standing modes}

GW produced at the interface by Reynolds stresses may travel across the entire stratified layer and reflect on the top boundary. The constructive interactions of propagating waves and their reflections excite and maintain standing modes $(\mathrm{SM})$, which are global resonances of the cavity formed by the stratified layer. As will be shown in section 4, numerous SM are excited, each one corresponding to a given wavelength and frequency. We mostly find SM with relatively small wavenumbers and/or relatively large frequencies, because they saturate at higher amplitude (see section 4).

As multiple SM coexist, it is difficult to isolate them visually. Sometimes, one mode dominates locally, appearing as a checkerboard-like pattern associated with well-defined wavelengths in the radial and horizontal directions, as visible on the bottom right part of figure 5 . Yet, most of the background is usually a mix of propagating waves and various SM (for example, see the backgrounds of the stratified layers in figures 3 left and 5). To distinguish between all contributions and analyze the properties of each wave separately, a spectral analysis, both in space and time, is necessary. This is the purpose of the next section.

\section{SPECTRAL PROPERTIES OF INTERNAL GRAVITY WAVES}

\section{$4.1(\ell, \omega)$ spectra}

The properties of GW can be characterized by their kinetic energy spectrum in both space and time, which indicates how much energy is contained in each spherical harmonics degree $\ell$ and frequency $\omega$. To obtain these spectra, we record the velocity components $V_{r}\left(r_{j}, \ell, m, t\right), V_{\theta}\left(r_{j}, \ell, m, t\right)$ and 
$V_{\phi}\left(r_{j}, \ell, m, t\right)$ at each time step and at 10 different radii $r_{j}(j=1$ to 10$)$ across the shell. Then, at each radius $r_{j}$, we compute the kinetic energy spectrum for each component,

$$
E_{r / \theta / \phi}\left(r_{j}, \ell, \omega\right)=\sum_{m} \operatorname{psd}\left[V_{r / \theta / \phi}\left(r_{j}, \ell, m, t\right)\right],
$$

where psd denotes the power spectrum density, calculated using the pwelch function of Matlab. The total kinetic energy spectrum is then

$$
E\left(r_{j}, \ell, \omega\right)=E_{r}\left(r_{j}, \ell, \omega\right)+E_{\theta}\left(r_{j}, \ell, \omega\right)+E_{\phi}\left(r_{j}, \ell, \omega\right) .
$$

Kinetic energy spectra $E\left(r_{j}, \ell, \omega\right)$ in $(\ell, \omega)$ space are plotted on figure 6 at four different radii, one in the convective region and the three others in the stratified layer, for simulation P1. Figure 6a shows that the convective flows that excite GW contain energy at a range of length scales and frequencies with, as expected for a turbulent flow in 3D, a decreasing amplitude when wavenumber and/or frequency increases. Yet, the spectral energy distribution in the stratified layer contains more complex structures, indicating that a large set of waves is excited (figure $6 \mathrm{p}, 6 \mathrm{c}$ and $6 \mathrm{~d}$ ). This shows that, even though the energy for the excitation of GW is injected by the convection, GW create their own dynamics inside the layer.

Whether or not the signal displayed on figures 6b-d represents GW can be confirmed by taking advantage of the geometric properties of internal waves. Campagne et al. (2015) then Savaro et al. (2020) showed that, when the kinetic energy is dominated by linear GW, we have

$$
\frac{E^{r}(\ell, \omega)}{E^{\perp}(\ell, \omega)}=\frac{\left(\omega^{*}\right)^{2}}{1-\left(\omega^{*}\right)^{2}}
$$

where the spectra $E^{r}(\ell, \omega)$ and $E^{\perp}(\ell, \omega)$ contain respectively the radial and horizontal components of the kinetic energy and $\omega^{*}=\omega / N$ is the frequency normalized by the Brunt-Väisälä frequency. Figure 7 shows the ratio $E^{r}(\ell, \omega) / E^{\perp}(\ell, \omega)$ averaged between $\ell=1$ and $\ell=140$ at $r=1.318$. The relation 25 is satisfied for frequencies between $0.4 N$ and $N$, which indicates that this portion of the spectra contains GW only. On the contrary, at frequencies $\omega<0.4 N$, there is a clear mismatch with relation 25 which indicates that the low-frequency part on figure 6 does not contain only linear GW and that most of its energy is non-linear GW patterns.

At each radius displayed, the GW lobe is bounded by the Brunt-Väisälä frequency (indicated 

by horizontal dashed lines in figures 6b-d), since higher frequencies waves are evanescent. This GW lobe is composed of a continuous background and a series of discrete ridges. Each of the discrete patches forming these ridges corresponds to a standing mode with specific $\ell$ and $\omega$. The ridges formed by these patches are visible at all radii across the stratified layer and each ridge corresponds to a given radial wavelength. At a given radius, a vertical cut at fixed degree $\ell$ of the $(\ell, \omega)$ spectrum gives the frequency profiles shown on figure $8 \mathrm{~b}$. These profiles show an overall decrease of the kinetic energy with $\omega$, superimposed by modes in the form of sharp peaks of kinetic energy, which are sometimes two orders of magnitude larger than the local background. Increasing the stiffness, i.e. the stratification strength, to $S=100$ leads to more numerous modes, especially in the low- $\ell$ high- $\omega$ part of the spectrum. In fact, increasing the stiffness creates a sharper, less deformable interface: the cavity formed by the stratified layer keeps a constant geometry over time, which facilitates resonances and modes formation. On the contrary, for lower values of the stiffness, the interface is more deformable. In that case, the cavity is less clearly defined which makes it harder for modes to form. However for the $S=100$ case, we observe that modes and propagating waves saturate at a comparatively much lower amplitude (figure 9).

These well-identifiable modes are present over a more diffuse background, which contains propagating waves. Figures $6 \mathrm{p}$ to $6 \mathrm{~d}$ show that, as the radius increases, the area covered by this background shrinks, particularly in the low- $\omega$, high- $\ell$ part of the lobe, as a result of the gradual attenuation of propagating waves when they progress upwards. Linear wave damping causes the wave energy flux $F$ to decrease exponentially with the distance to the interface $\left(r=r_{\text {int }}\right)$ as

$$
F(r)=F\left(r_{\text {int }}\right) e^{-2 \gamma\left(r-r_{\text {int }}\right)},
$$

where

$$
\gamma=\frac{(1+P r)}{2} k_{\perp}^{3} N^{3} / \omega^{4}
$$

is the waves decay rate with $k_{\perp}$ the horizontal wavenumber. In that case, for fixed $N, \lambda=(2 \gamma)^{-1}$ is the typical attenuation length for propagating waves, that depends only on $\omega$ and $k_{\perp}$. In spherical geometry, $k_{\perp}=r^{-1}[\ell(\ell+1)]^{1 / 2}$, which has a dependence on the radius. In addition, $N$ varies slightly with $r$ in our model. Therefore, here $\gamma$ is a function of $\omega, \ell$ and $r$. A GW with degree $\ell$ and 
frequency $\omega$ sees an effective decay rate

$$
\gamma_{\mathrm{eff}}(\ell, \omega)=\frac{1}{r_{o}-r_{\mathrm{int}}} \int_{r_{\mathrm{int}}}^{r_{o}} \gamma(r) \mathrm{d} r=[\ell(\ell+1)]^{3 / 2} \omega^{-4} \phi,
$$

where

$$
\phi=\frac{1}{r_{o}-r_{\text {int }}} \int_{r_{\text {int }}}^{r_{o}} r^{-3} N^{3}(r) \mathrm{d} r .
$$

Therefore, the effective attenuation length is

$$
\lambda_{\mathrm{eff}}(\ell, \omega)=\frac{1}{2 \gamma_{\mathrm{eff}}}=\frac{1}{2} \phi^{-1}[\ell(\ell+1)]^{-3 / 2} \omega^{4} .
$$

Hence, in the $(\ell, \omega)$ spectral space, iso- $\lambda_{\text {eff }}$ lines are defined by

$$
\omega=\left(2 \lambda_{\mathrm{eff}} \phi\right)^{1 / 4}[\ell(\ell+1)]^{3 / 8} .
$$

Three iso- $\lambda_{\text {eff }}$ lines defined by equation (31) are represented in the $(\ell, \omega)$ spectra in figures $6 \mathrm{~b}-\mathrm{d}$. Immediately above the interface (figure 6b), numerous propagating waves are present, with various length scales, frequencies and intensities, depending on how they were excited. When $r$ increases, the waves associated to the smallest attenuation depth disappear, while the less attenuated waves remain. This is well visible on figures $6 \mathrm{~b}-\mathrm{d}$ in which the waves signal located below a line of constant $\lambda_{\text {eff }}$ progressively disappear when $r$ increases. When the attenuation length $\lambda_{\text {eff }}$ reaches a critical value compared to the thickness of the layer, propagating waves above the iso- $\lambda_{\text {eff }}$ line can reach the top of the layer, reflect on the outer boundary and eventually form modes by constructing interferences at selected resonance values in radial structure. In that case, the iso- $\lambda_{\text {eff }}$ line (the dashed-dotted line on figures 6p-d) separates approximately propagating waves (below the line) from modes (above the line). Equation (27) indicates that high $\omega$, low $\ell$ waves are comparatively less attenuated. This is the reason why modes are found in the top left part of the waves lobe in the $(\ell, \omega)$ spectra. The saturation amplitude of modes depends both on the attenuation of propagating waves and on their excitation by the convection. Propagating waves with a higher excitation energy and/or a smaller decay rate tend to form more energetic modes.

To separate the contributions of propagating waves and standing modes in the spectra, we apply a damping term in the top part of the stratified layer as described in equation (2) (simulation P1d). This damping term replaces the top rigid boundary by a region across which waves rapidly vanish. 
By preventing wave reflection off the top boundary this term eliminates most standing modes. The introduction of a damping term at the top of the stable layer is not motivated by physical arguments for application to cores bounded by a rigid mantle, it is simply a numerical trick that allows us to remove standing modes and isolate the contribution of propagating waves in the spectra. Figure 10 shows $(\ell, \omega)$ spectra of the kinetic energy for simulations P1 (without damping, figure 10a) and P1d (with damping, figure 10p). The damped spectrum confirms that the diffuse background is produced by propagating waves and that the discrete ridges are the traces of standing modes.

To compare the energy contained in standing modes versus propagating waves, we integrate the $(\ell, \omega)$ spectra with and without the damping term in the top part of the layer. The total energy of waves (propagating waves and standing modes) is

$$
E_{\mathrm{tot}}\left(r_{j}\right)=\sum_{\ell} \int_{\omega} E\left(r_{j}, \ell, \omega\right) \mathrm{d} \omega .
$$

The energy of propagating waves can be estimated by integrating the spectra obtained with the damping term at the top of the layer:

$$
E_{\mathrm{PW}}\left(r_{j}\right) \approx \sum_{\ell} \int_{\omega} E_{\mathrm{damped}}\left(r_{j}, \ell, \omega\right) \mathrm{d} \omega .
$$

Note that this assumes that all modes have been effectively removed by the damping term, which in practice is difficult to fully ensure. The energy contained in standing modes is the total energy minus the energy of propagating waves:

$$
E_{\mathrm{SM}}\left(r_{j}\right)=E_{\mathrm{tot}}\left(r_{j}\right)-E_{\mathrm{PW}}\left(r_{j}\right) .
$$

We then compute the ratio

$$
R_{\mathrm{SM}}\left(r_{j}\right)=E_{\mathrm{SM}}\left(r_{j}\right) / E_{\mathrm{tot}}\left(r_{j}\right),
$$

which estimates the fraction of the total energy contained in standing modes. Marginally above the interface, at $r=1.046$, we find that $R_{\mathrm{SM}}\left(r_{j}\right)$ is close to $21 \%$, which indicates that propagating waves account for most of the energy above the interface. Further up, at $r=1.136$ and $r=1.227$, the ratio rises to respectively $R_{\mathrm{SM}}\left(r_{j}\right) \approx 59 \%$ and $R_{\mathrm{SM}}\left(r_{j}\right) \approx 83 \%$. This is because propagating waves are gradually attenuated whereas the magnitude of modes is mostly the same throughout 
the layer. This shows that, while propagating waves dominate the signal immediately above the interface, modes account for most of the total waves energy higher in the stratified layer.

The spectra from figure 6 can be confronted to the theoretical predictions of Lecoanet \& Quataert (2013). That work used the same assumptions as Goldreich \& Kumar (1990): i) waves are excited by Reynolds stresses associated with turbulent convective eddies; ii) the flow can be decomposed into eddies, the excitation can be calculated for each eddy individually and the total wave generation is given by summing over all eddies; iii) the eddies of size $h$ have velocities which scale like $h^{1 / 3}$ and stay coherent for their turnover time. Put together, these predict the kinetic energy spectrum

$$
K E \sim \rho_{0}\left(\omega_{c} H\right)^{2}\left(\ell \frac{H}{r}\right)^{5}\left(\frac{\omega}{\omega_{c}}\right)^{-17 / 2} .
$$

This theory is only applicable for moderately low $\ell$ and high $\omega$ : at high $\ell$ and low $\omega$, damping will dominate and cause the kinetic energy to drop rapidly. Figure 8 a shows $\ell$-spectra for two different frequencies and at two different radii for simulation P1. We include a $\ell^{5}$ power law as predicted by the theory for the energy increase at low values of $\ell$ which is in good agreement with the curves from the simulation. Similarly, figure $8 \mathrm{p}$ displays $\omega$-spectra for $\ell=2$ and $\ell=6$ at two different radii for simulation P1, showing a very rapid drop in the kinetic energy when increasing the frequency up to $\omega \sim 10^{5}$. We include a $\omega^{-8.5}$ power law scaling as predicted by the theory. Here as well, the agreement with the theory is rather satisfactory. This suggests that the Reynolds stress mechanism is indeed at play in our simulations. Note that the peaks that superimpose to the average curves are caused by standing modes, which are not included in the theory. Furthermore, since this theory is based on (i) the assumption that Reynolds stresses are the main source of GW and (ii) a simple model of turbulence based on a Kolmogorov type turbulence, this agreement opens new perspectives for developing predictive tools of $\mathrm{GW}$ in more relevant (e.g. rotating) configurations.

\subsection{Effect of the Prandtl number}

Figure 11 shows $(\ell, \omega)$ spectra for simulations P03, P1 and P3, which have a comparable convective forcing but differ by their Prandtl numbers (respectively $\operatorname{Pr}=0.3, \operatorname{Pr}=1$ and $\operatorname{Pr}=3$ ). 
Although the variation in the Prandtl number is modest, we observe dramatic changes in the spectra. For simulation $\operatorname{Pr}=0.3$, all the energy is confined to $\ell \lesssim 70$, while higher Prandtl number simulations have energy up to higher spherical harmonics degrees (up to $\ell \sim 120-130$ for $\operatorname{Pr}=3)$. The $\operatorname{Pr}=0.3$ simulation also appears to have more energetic modes compared to the larger Prandtl number simulations and contains more energy in the low- $\omega$, low- $\ell$ part of the spectrum.

Interpreting these observations is not straightforward since both the excitation and the attenuation of waves may vary when changing the Prandtl number. The attenuation of waves increases with the Prandlt number (see equation (27)) because the viscous damping is stronger. As a consequence of this stronger attenuation, modes saturate at a lower amplitude for higher Prandtl numbers. This explains the lower amplitudes of modes in the $\operatorname{Pr}=3$ simulation compared to the $\operatorname{Pr}=0.3$ simulation. Note that the study of Couston et al. (2018b) showed that, in a Cartesian box, the QBO is obtained for low Prandtl numbers and strengthens when the Prandtl number decreases.

Such differences observed for relatively modest variations of the Prandtl number suggest that the wave dynamics of a thermally stratified layer $(\operatorname{Pr} \sim 0.1)$ could be very different from that of a chemically stratified layer $\left(S c>10\right.$, where $S c=\nu / \kappa^{\xi}$ is the Schmidt number, the equivalent of the Prandtl number for composition, with $\kappa^{\xi}$ the compositional molecular diffusivity). In a thermally stratified layer, we expect the energy to be concentrated in fewer but more energetic modes, whereas a chemically stratified layer could host more numerous but weaker modes up to high spherical harmonics degrees. We also expect a thermally stratified layer to be more prone to the development of a QBO-like mean flow than a chemically stratified layer. More generally, our results show that important aspects of the dynamics of the core may be missed when considering only the $\operatorname{Pr}=1$ simulation, as is classically done in numerical studies of the outer core. These conjectures need to be confirmed by running simulations including rotational and magnetic effects. 


\section{ENERGY TRANSFER}

How much energy is transfered from the convective region to GW in a stratified layer is an important question for the evolution of the Earth's core and to assess the effect of the layer on the gravity field. The global energy transfer can be measured by the ratio of the mean kinetic energy in the stratified and convective regions,

$$
R=\frac{\left\langle E_{\text {strat }}\right\rangle}{\left\langle E_{\text {conv }}\right\rangle}=\frac{\frac{1}{V_{\text {sup }}} \int_{r=r_{\text {int }}+\delta}^{r_{o}}\langle E(r)\rangle r^{2} d r}{\frac{1}{V_{\text {inf }}} \int_{r=r_{i}}^{r_{\text {int }}-\delta}\langle E(r)\rangle r^{2} d r},
$$

where $E(r)$ is the horizontally averaged kinetic energy at radius $r$ and $r_{\text {int }}$ is the interface radius. Brackets \langle\rangle denote time averages. The interface region has been excluded by setting $\delta=0.025$, so that the region of rapidly dropping kinetic energy between the convective and stratified regions is not considered. For simulation $\mathrm{P} 1(\operatorname{Pr}=1)$ we find $R \sim 1.6 \times 10^{-3}$, i.e. the rms velocity has decreased by a factor $\sim 25$. We find a similar ratio $R \sim 1.2 \times 10^{-3}$ for simulation $\mathrm{P} 03(\operatorname{Pr}=0.3)$, and $R \sim 1.4 \times 10^{-3}$ for simulation P3 $(\operatorname{Pr}=3)$. Note that these ratios are obtained with the Rayleigh number and stiffness adopted in our simulations. They may change significantly when moving towards more realistic parameters (i.e. higher Rayleigh number with rotational effects). They may also depend on the choice of the parameter $\delta$ in equation (37), on the stratification strength and on whether the stratification is thermal (low Prandtl number) or chemical (high Schmidt number).

These global ratios mask the fact that the kinetic energy in the stratified layer is a strong function of radius, wavelength and frequency. Figure 12 a shows the time average radial profiles of the angle-integrated kinetic energy for simulation P1 $(\operatorname{Pr}=1)$. In the convective region, the kinetic energy is fairly stable around $\left\langle E_{\text {conv }}\right\rangle \sim 2-3 \times 10^{6}$. Within the first $15 \%$ of the layer above the interface, the kinetic energy drops by almost 3 orders of magnitude, so that the rms velocity is about 30 times less than the rms velocity in the convective region. Then, the kinetic energy further drops by about 2 orders of magnitude when moving upwards in the stratified layer, until the rms velocity in the outermost portion of the layer has droped by a factor $\sim 250$ compared with the convective region. These factors are consistent with the rescaling factor of $43-100$ that we had to apply on velocities in the stratified layers on figures 3 to 5 to visualize velocities both in the convective region and in the stratified layer using the same colorbar. For a given horizontal 
wavenumber $k_{\perp}$, the theory of Lecoanet \& Quataert (2013) finds that the kinetic energy decrease follows a power law,

$$
E\left(r, k_{\perp}\right) \sim\left[(1+\operatorname{Pr}) N k_{\perp}^{3}\left(z-z_{\text {int }}\right)\right]^{-17 / 8},
$$

where $z_{\text {int }}$ is the radius of the interface. In spherical geometry, $k_{\perp}=\sqrt{\ell(\ell+1)} / r$. Furthermore, in our case $N$ depends slightly on the radius. Therefore we have

$$
\langle E(r)\rangle \sim\left[N(r) \frac{r-r_{\text {int }}}{r^{3}}\right]^{-17 / 8} .
$$

This theoretical law is reported as a dashed line on figure $12 \mathrm{a}$. We observe that the kinetic energy decays more slowly in our simulations compared to the theoretical prediction. We suggest that this lower decrease is due to the presence of numerous modes in our simulations, as these are attenuated less rapidly with the radius and are not considered in the theory of Lecoanet \& Quataert (2013).

As already shown on the spectra of figure 6, the energy distribution at each radius depends on the spherical harmonic degree $\ell$ and frequency $\omega$. Figure $12 \mathrm{~b}$ shows the time averaged $\ell$-spectra of the kinetic energy in each half space (excluding again the interface region $r_{\text {int }} \pm \delta$ ). The spectrum in the stratified layer has been normalized by the $\ell=1$ value of the convective spectrum, to better visualize the relative drop as a function of $\ell$. Like in figure 6, the spectrum in the stratified layer shows that the low $\ell$ components have a higher kinetic energy, with a peak at $\ell=2$, and that these degrees are better transmitted from the convective region compared with higher $\ell$ s.

Figure 13 shows the kinetic energy ratio between the convective region and the stratified layer, at different radii. As was already visible on the spectra of figure 6, not all convective frequencies and wavelengths are transmitted to the stratified layer. Convection injects energy at all scales, but the energy distribution in the layer depends on the waves dynamics. We also note that the kinetic energy fraction of standing modes is nearly constant across all radii, which is expected since their amplitude is approximately constant throughout the stratified layer.

In the next section, we use the results above on the energy distribution and typical velocities in the stratified layer to evaluate orders of magnitude for the waves-induced perturbations of the gravity field. 


\section{GEOPHYSICAL SIGNATURES IN THE GRAVITY FIELD}

GW produce small deviations from the mean density profile of the stratified layer, as well as pressure fluctuations at the CMB. These anomalies of density and pressure induce small perturbations in the gravity field of the Earth, which may be detected in gravity data. Previous attempts to detect the gravitational signatures of inertial waves - another type of waves that develop in the whole rotating outer core - remained unsuccessful (Melchior \& Ducarme, 1988). This was due to the lower sensitivity of gravimeters at the time and to the fact that the main frequencies of inertial waves coincide with stronger signals from tides or other geophysical phenomena. By contrast, the spectra from figure 6 6 reveal that GW in a stratified layer atop Earth's core may exist over a wide range of frequencies and wavelengths, some of which may be distinct from any other geophysical signal. In addition, modern superconducting gravimeters, which are sensitive to fluctuations of only a few nGal or less at the Earth's surface (Rosat \& Hinderer, 2018), now offer more hope for the detection of GW. In the following lines, we estimate an order of magnitude for the gravity perturbations induced by GW at the Earth's surface, and compare it to the sensitivity of presentday gravimeters. Since we aim at obtaining orders of magnitude, we will focus on gravity changes induced by density perturbations and will neglect elastic deformations caused by pressure fluctuations at the $\mathrm{CMB}$, as these are mostly corrections and are negligible for $\ell>2$ (Dumberry, 2010). Note that we write the calculations for the non-rotating and non-magnetic case here, but the same procedure can be followed for $\mathrm{GW}$ in a rotating core with a magnetic field.

In geodesy, the variation of gravitational potential $\Delta V(r, \theta, \phi)$ at a radius $r$ above the Earth's surface is usually expanded on a spherical harmonics basis,

$$
\Delta V(r, \theta, \phi)=\frac{G M}{r} \sum_{\ell=2}^{\infty} \sum_{m=0}^{\ell}\left(\frac{r_{e}}{r}\right)^{\ell} \mathcal{V}_{\ell m},
$$

where $M$ is the mass of the Earth, $r_{e}$ the Earth's radius and

$$
\mathcal{V}_{\ell m}=\left(C_{\ell m} \cos m \phi+S_{\ell m} \sin m \phi\right) R_{\ell m} P_{\ell m}(\cos \theta)
$$

where $P_{\ell m}(\cos \theta)$ are the Legendre polynomials with the $4 \pi$ normalization factor

$$
R_{\ell m}=\sqrt{\left(2-\delta_{m, 0}\right)(2 \ell+1) \frac{(\ell-m) !}{(\ell+m) !}} .
$$


Note that we do not consider degrees $\ell=0$ and $\ell=1$ in the above summation because we assume that mass is conserved and that core motions do not change the location of the centre of mass. In (41), $C_{\ell m}$ and $S_{\ell m}$ are referred to as the Stokes coefficients. Perturbations of the gravity field are usually measured in terms of changes in these coefficients. Typical changes $\Delta C$ in the Stokes coefficients caused by density perturbations $\delta \rho$ in the core can be estimated by (Dumberry, 2010)

$$
\Delta C \sim \frac{4 \pi}{(2 \ell+1)^{3 / 2}}\left(\frac{r_{o}}{r_{e}}\right)^{\ell} \frac{r_{o}^{2} h \delta \rho}{M},
$$

where $r_{o}$ is the radius of the outer core and $h$ the thickness of the region across which the density perturbations extend. Here, $h$ is the thickness of the stratified layer at the top of the core. The typical density perturbation $\delta \rho$ induced by a GW of frequency $\omega$ and vertical velocity $W$ can be estimated using the GW polarisation relation (Sutherland, 2010)

$$
i \frac{\omega g \delta \rho}{\rho_{0}}+N^{2} W=0
$$

where $g$ is the gravitational acceleration at the top of the core, which leads to

$$
\delta \rho \sim \frac{\rho_{0} N^{2} W}{\omega g}
$$

Injecting (45) into (43) gives typical variations $\Delta C$ of the Stokes coefficient of order

$$
\Delta C \sim \frac{4 \pi}{(2 \ell+1)^{3 / 2}}\left(\frac{r_{o}}{r_{e}}\right)^{\ell} \frac{r_{o}^{2} h \rho_{0} N^{2} W}{M \omega g} .
$$

From equation (43), it is clear that changes in the Stokes coefficients decrease very rapidly with increasing $\ell$, by typically an order of magnitude for an increase of two in $\ell$. Therefore, only waves with sufficiently low $\ell$ have a chance to produce a detectable signature. Since propagating waves are essentially local processes and typically involve large $\ell$ s and various frequencies, they will be hard to detect. On the contrary, low- $\ell$ modes are better candidates, because they resonate globally in the entire stratified layer with a well-defined frequency. Low- $\ell$ modes also are the most energetic, particularly at low Prandtl number (figures 6, 11 and 12).

The spectra in figure 6 show that low- $\ell$ modes are found at frequencies significantly lower than $N$, typically $\omega \sim 0.2 N$. In the literature, the value of $N$ is often expressed through its squared ratio $(N / \Omega)^{2}$ to the Earth's rotation frequency $\Omega$. A range of values has been proposed for this ratio in the literature. Geomagnetic observations require a ratio of order 1 (Buffett, 2014; Buffett et al., 
2016) while seismic observations favor larger values (Helffrich \& Kaneshima, 2010), giving the range

$$
\left(\frac{N}{\Omega}\right)^{2} \in[0.5-600]
$$

Assuming $\omega \sim 0.2 N$ and $\Omega=7.292 \times 10^{-5} \mathrm{~s}^{-1}$, we obtain typical GW periods comprised between $5 \mathrm{~h}$ and $170 \mathrm{~h}$.

Eventually, the evaluation of $\Delta C$ requires a value for the typical radial velocity $W$ of the waves. This can be evaluated from the kinetic energy ratios calculated in equation (37) and discussed in section 5 . These ratios give a mean drop of kinetic energy of $\sim 10^{-3}$ between the convective region and the waves, which leads to typical wave velocities that are about 30 times as small as the convective rms velocity. Alternatively, $W$ can be roughly estimated for the low- $\ell$ modes (which we focus on here) by looking at the bottom left corner of figure 13. This figure predicts kinetic energy ratios comprised between $10^{-2}$ and $10^{-1}$ for these modes, that is a velocity drop of only a factor 3-10. We therefore adopt a conservative factor 20 for the drop in kinetic energy for the low- $\ell$ modes. We note that these ratios may not be representative of realistic conditions in the Earth's core but here we aim at deriving a general estimation method. The calculations hereafter can be performed using kinetic energy ratios obtained with more realistic simulations including rotation and magnetic field. Taking $5 \times 10^{-4} \mathrm{~m} \mathrm{~s}^{-1}$ as the typical velocity in the convecting region (Christensen \& Aubert, 2006; Finlay \& Amit, 2011), we estimate $W \sim 2.5 \times 10^{-5} \mathrm{~m} \mathrm{~s}^{-1}$. For the other numerical values, we adopt $g=10 \mathrm{~m} \mathrm{~s}^{-2}, M=5.97 \times 10^{24} \mathrm{~kg}, \rho_{0}=9.903 \times 10^{3} \mathrm{~kg} \mathrm{~m}^{-3}$, $\Omega=7.292 \times 10^{-5} \mathrm{~s}^{-1}, r_{o}=2.891 \times 10^{6} \mathrm{~m}, h=100 \mathrm{~km}$ and $r_{e}=6.371 \times 10^{6} \mathrm{~m}$. Figure 14 displays the estimates for the changes in the Stokes coefficients obtained from equation (46) with the numerical values given above, for $\ell=2$ to 10 and for the two end-member values of the $(N / \Omega)^{2}$ ratio (0.5 and 600). These typical changes in the Stokes coefficients should be compared to the accuracy of superconducting gravimeters, which is around $1 \mathrm{nGal}$ (Rosat \& Hinderer, 2018). The gravity variation $\Delta g$ at the Earth's surface is the radial derivative of $\Delta V$ at $r=r_{e}$, that is

$$
\Delta g=\frac{G M}{r_{e}^{2}} \sum_{\ell=2}^{\infty} \ell \sum_{m=0}^{\ell}\left(\Delta C_{\ell m} \cos m \phi+\Delta S_{\ell m} \sin m \phi\right) R_{\ell m} P_{\ell m}(\cos \theta) .
$$

The above equation can then be used to estimate the detection limit on $\Delta C$ for $\Delta g \sim 1 \mathrm{nGal}$. For 
example, for $\ell=m=2$ and $\Delta g=1 \mathrm{nGal}$, the minimal detectable change in the Stokes coefficient is $\Delta C_{22}=\Delta g r_{e}^{2} /\left(G M \ell R_{22} P_{22}(\cos \theta)\right) \sim 2.5 \times 10^{-13}$ at the equator $(\theta=\pi / 2)$. The minimal detectable perturbation of the Stokes coefficients at the equator, for $\Delta g=1 \mathrm{nGal}$ and $m=\ell$ is represented by a black dashed line on figure 14. This line shows that, for strong stratifications, several modes (possibly up to $\ell=8$ ) could potentially be observed. For weaker stratifications, only the $\ell=2$ mode would have a chance to be detected. Besides, GW modes in a stratified layer have typical frequencies comprised between $\sim 1$ hour to a few hundreds of hours, depending on the stratification strength $N$. Such frequencies may be distinct from tides or other geophysical phenomena. However, note that the above orders of magnitude depend on the typical velocity of gravity waves, which we have estimated from our non-rotating, non-magnetic, simulations, with control parameters far from realistic values. The typical wave velocity and hence the typical density anomaly and induced gravity signal of GW may change significantly in core conditions, so that the perturbations in the Stokes coefficients derived in the previous calculations may vary significantly when more geophysical ingredients are included. To conclude more robustly on the detectability of GW in the gravity data, a (much more costly) systematic study would be necessary to obtain scaling laws.

\section{SUMMARY AND DISCUSSION}

In this study, we performed numerical simulations of a two-layer, non-rotating, non-magnetic, spherical shell, in which a stratified layer lies on top of a turbulent convective region. This configuration leads to the development of internal gravity waves $(\mathrm{GW})$ in the stratified layer, for which gravity is the restoring force. Reynolds stresses generate GW that transport energy away from the interface. While they progress upwards, propagating waves are linearly damped. Waves with smaller horizontal wavenumbers and/or relatively large frequencies are comparatively less attenuated. These waves can make it to the top of the layer and reflect on the outer boundary. The constructive interactions of propagating waves and their reflections on the top boundary excite and maintain standing modes that resonate in the cavity formed by the stratified layer. Analysis of wavenumber-frequency spectra reveals that the wave energy is distributed among propagating 
waves and standing modes across wavelengths and frequencies. While energy is injected in the stratified layer by convective motions, the energy distribution in the layer differs from that of the convective region and depends on the waves' own dynamics. In the non-rotating and non-magnetic case, we observe significant differences in the spectral energy distribution of the waves when only modestly changing the Prandtl numbers, even if total wave energy does not change much. This result suggests that one may miss important aspects of the dynamics of the core when fixing $\operatorname{Pr}=1$, as is done in most studies for numerical convenience. It also suggests that thermally and compositionally stratified layers may host GW with very different energy distributions. Overall, for the control parameters considered in this study, we find that the average kinetic energy in the stratified layer is about $0.1 \%$ that of the convective region; that is, the typical waves velocity is about 30 times smaller than the rms convective velocity. Based on this typical wave velocity, we find that signatures of GW could potentially be detected in gravimetric data. However, the typical waves velocity, upon which these results are based, may change when moving towards more realistic control parameters and including rotational and magnetic effects. The wave dynamics may also depend on the thickness of the stratified layer, which is unrealistically large in our simulations. Therefore, the figures derived from the non-rotating simulations in this paper should be interpreted very cautiously, as they may not be relevant values for the core. To draw robust conclusions, it is necessary to perform a more systematic exploration of the parameter space including rotational and magnetic effects.

Although this study neglects rotational and magnetic effects, it sets the conceptual bases and tools necessary to analyse the properties of internal waves in a stratified layer and the perturbations these waves might induce on the gravity field. Therefore, it is a first step towards more geophysically realistic simulations. It also confirms the relevance of modelling the stratified layer using a non-linear equation of state. Future works must include the effects of rotation and magnetic field as these are key ingredients of core dynamics. In order to excite waves with a rich convective spectrum, costly simulations at high Reynolds number and subsequently low Ekman number are necessary. Since both rotation and magnetic fields break the isotropy of the system, several differences may appear with respect to the non-rotating case. First, as rotationally-dominated flows 
are strongly anisotropic, taking the form of columnar convection elongated along the rotation axis, internal waves may be excited to very different amplitudes as a function of colatitude. Hence, the total and latitudinal energy transfer from the convective region to the waves is likely to be affected. Second, while propagating waves and modes may still coexist in the layer, they are likely to be modulated by rotational effects and further damped by Ohmic dissipation. Furthermore, additional types of waves such as MAC waves, magnetic Rossby waves and Alfvén waves may be present in the layer (Braginsky, 1999). We anticipate that, to reach the Earth's surface, magnetic waves will need to have sufficiently long periods in order to pass the skin effect exerted by the not perfectly insulating mantle. Eventually, symmetry breaking by rotation and magnetic field may lead to the formation of large-scale mean zonal flows in the stratified layer, with various dynamical impacts (Rogers et al., 2013). In all cases, the tools described in the present paper can also be used to study the dynamics of internal waves in the presence of rotation and magnetic field, and to relate it to observed geophysical quantities.

\section{DATA AVAILABILITY}

The data underlying this article will be shared on reasonable request to the corresponding author.

\section{ACKNOWLEDGMENTS}

We thank W. Dietrich and an anonymous reviewer for their valuable comments that were very helpful to improve the quality of this manuscript. We also thank S. Rosat and F. Chambat for useful help and discussions on determining the gravimetric signatures of GW. Calculations were performed on the Cobra cluster of the MPCDF facility and on the Jean Zay CSL cluster (IDRIS, Orsay, France) under project number i2015047370. The authors acknowledge funding by the European Research Council under the European Union's Horizon 2020 research and innovation program through Grant No. 681835-FLUDYCO-ERC-2015-CoG. 


\section{REFERENCES}

Aerts, C., Christensen-Dalsgaard, J., \& Kurtz, D. W., 2010. Asteroseismology, Springer Science \& Business Media.

Alvan, L., Brun, A. S., \& Mathis, S., 2014. Theoretical seismology in 3D: nonlinear simulations of internal gravity waves in solar-like stars, Astron. Astrophys., 565, A42.

André, Q., Barker, A. J., \& Mathis, S., 2017. Layered semi-convection and tides in giant planet interiors-I. Propagation of internal waves, Astron. Astrophys., 605, A117.

Arveson, S. M., Deng, J., Karki, B. B., \& Lee, K. K., 2019. Evidence for Fe-Si-O liquid immiscibility at deep Earth pressures, PNAS, 116(21), 10238-10243.

Baldwin, M. P., Gray, L. J., Dunkerton, T. J., Hamilton, K., Haynes, P. H., Randel, W. J., ... \& Jones, D. B. A., 2001. The quasi-biennial oscillation, Rev. Geophys., 39(2), 179-229.

Bouffard, M., Choblet, G., Labrosse, S., \& Wicht, J., 2019. Chemical convection and stratification in the Earth's outer core, Front. Earth Sci., 7, 99.

Bouffard, M., Landeau, M., \& Goument, A., 2020. Convective erosion of a primordial stratification atop Earth's core, Geophys. Res. Lett., 47(14), e2020GL087109.

Braginsky, S. I., 1999. Dynamics of the stably stratified ocean at the top of the core, Phys. Earth Planet. Inter, 111(1-2), 21-34.

Buffett, B., 2014. Geomagnetic fluctuations reveal stable stratification at the top of the Earth's core, Nature, 507(7493), 484-487.

Buffett, B., \& Seagle, C. T., 2010. Stratification of the top of the core due to chemical interactions with the mantle, J. Geophys. Res. Solid Earth, 115(B4).

Buffett, B., Knezek, N., \& Holme, R., 2016. Evidence for MAC waves at the top of Earth's core and implications for variations in length of day, Geophys. J Int., 204(3), 1789-1800.

Campagne, A., Gallet, B., Moisy, F., \& Cortet, P. P., 2015. Disentangling inertial waves from eddy turbulence in a forced rotating-turbulence experiment, Phys. Rev. E, 91(4), 043016.

Charbonnel, C., \& Talon, S., 2005. Influence of gravity waves on the internal rotation and Li abundance of solar-type stars, Science, 309(5744), 2189-2191.

Christensen, U. R., 2006. A deep dynamo generating Mercury's magnetic field, Nature, 444(7122), 10561058 .

Christensen, U. R., 2018. Geodynamo models with a stable layer and heterogeneous heat flow at the top of the core, Geophys. J Int., 215(2), 1338-1351.

Christensen, U. R., \& Aubert, J., 2006. Scaling properties of convection-driven dynamos in rotating spherical shells and application to planetary magnetic fields, Geophys. J Int., 166(1), 97-114.

Christensen, U. R., \& Wicht, J., 2008. Models of magnetic field generation in partly stable planetary cores: Applications to Mercury and Saturn, Icarus, 196(1), 16-34. 
Couston, L. A., Lecoanet, D., Favier, B., \& Le Bars, M., 2017. Dynamics of mixed convective-stablystratified fluids, Phys. Rev. Fluids, 2(9), 094804.

Couston, L. A., Lecoanet, D., Favier, B., \& Le Bars, M., 2018. The energy flux spectrum of internal waves generated by turbulent convection, J. Fluid Mech., 854.

Couston, L. A., Lecoanet, D., Favier, B., \& Le Bars, M., 2018. Order out of chaos: Slowly reversing mean flows emerge from turbulently generated internal waves, $P R L, \mathbf{1 2 0}(24), 244505$.

Couston, L. A., Lecoanet, D., Favier, B., \& Le Bars, M., 2020. Shape and size of large-scale vortices: A generic fluid pattern in geophysical fluid dynamics, Phys. Rev. Res., 2(2), 023143.

Crossley, D. J., \& Rochester, M. G., 1980. Simple core undertones, Geophys. J Int., 60(2), 129-161.

Dintrans, B., Rieutord, M., \& Valdettaro, L., 1999. Gravito-inertial waves in a rotating stratified sphere or spherical shell, J. Fluid Mech., 398, 271-297.

Dormy, E., 1997. Modlisation numrique de la dynamo terrestre, Doctoral dissertation, Institut de physique du globe (Paris).

Dumberry, M., 2010. Gravity variations induced by core flows, Geophys. J Int., 180(2), 635-650.

Edelmann, P. V. F., Ratnasingam, R. P., Pedersen, M. G., Bowman, D. M., Prat, V., \& Rogers, T. M., 2019. Three-dimensional simulations of massive stars. I. Wave generation and propagation, ApJ, 876(1), 4.

Evonuk, M., \& Samuel, H., 2012. Simulating rotating fluid bodies: When is vorticity generation via densitystratification important?, Earth Planet. Sci. Lett., 317, 1-7.

Finlay, C. C., \& Amit, H., 2011. On flow magnitude and field-flow alignment at Earth's core surface, Geophys. J Int., 186, 175-192.

Friedlander, S., 1987. Hydromagnetic waves in the Earth's fluid core, Geophys. Astrophys. Fluid Dyn., 39(4), 315-333.

Fuller, J., Lecoanet, D., Cantiello, M., \& Brown, B., 2014. Angular momentum transport via internal gravity waves in evolving stars, $A p J, \mathbf{7 9 6}(1), 17$.

Gastine, T., Aubert, J., \& Fournier, A., 2020. Dynamo-based limit to the extent of a stable layer atop Earth's core, Geophys. J Int., 222(2), 1433-1448.

Gillet, N., Jault, D., Canet, E., \& Fournier, A., 2010. Fast torsional waves and strong magnetic field within the Earth's core, Nature, 465(7294), 74-77.

Goldreich, P., \& Kumar, P., 1990. Wave generation by turbulent convection. ApJ, 363(2), 694-704.

Gubbins, D., \& Davies, C. J., 2013. The stratified layer at the core-mantle boundary caused by barodiffusion of oxygen, sulphur and silicon, Phys. Earth Planet. Inter., 215, 21-28.

Helffrich, G., \& Kaneshima, S., 2010. Outer-core compositional stratification from observed core wave speed profiles, Nature, 468, 807-810.

Horst, L., Edelmann, P. V. F., Andrssy, R., Rpke, F. K., Bowman, D. M., Aerts, C., \& Ratnasingam, R. P., 
2020. Fully compressible simulations of waves and core convection in main-sequence stars, $A \& A, \mathbf{6 4 1}$, A18.

Jacobson, S. A., Rubie, D. C., David, C., Hernlund, J., Morbidelli, A., \& Nakajima, M., 2017. Formation, stratification, and mixing of the cores of Earth and Venus, Earth Planet. Sci. Lett., 474, 375-386.

Jaupart E., \& Buffett B., 2017. Generation of MAC waves by convection in Earth's core, Geophys. J Int., 209(2), 1326-1336.

Kaneshima, S., 2018. Array analyses of SmKS waves and the stratification of Earth's outermost core, Phys. Earth Planet. Inter., 276, 234-246.

Kaneshima, S., \& Helffrich, G., 2013. Vp structure of the outermost core derived from analysing large-scale array data of SmKS waves, Geophys. J Int., 193, 1537-1555.

Kaneshima, S., \& Matsuzawa, T., 2015. Stratification of Earth's outermost core inferred from SmKS array data, Prog. Earth Planet. Sci., 2, 15.

Landeau, M., Olson, P., Deguen, R., Hirsh, B. H, 2016. Core merging and stratification following giant impact, Nat. Geosci, 9(10), 786-789.

Laneuville, M., Hernlund, J., Labrosse, S., \& Guttenberg, N., 2018. Crystallization of a compositionally stratified basal magma ocean, Phys. Earth Planet. Inter., 276, 86-92.

Labrosse, S., 2015. Thermal evolution of the core with a high thermal conductivity, Phys. Earth Planet. Inter., 247, 36-55.

Léard, P., Favier, B., Le Gal, P., \& Le Bars, M., 2020. Coupled convection and internal gravity waves excited in water around its density maximum at $4^{\circ} \mathrm{C}$, Phys. Rev. Fluids, 5(2), 024801.

Lecoanet, D., \& Quataert, E., 2013. Internal gravity wave excitation by turbulent convection, MNRAS, 430(3), 2363-2376.

Lecoanet, D., Le Bars, M., Burns, K. J., Vasil, G. M., Brown B. P., Quataert, E., \& Oishi, J. S., 2015. Numerical simulations of internal wave generation by convection in water, $P R E, 91(6), 063016$.

Le Bars, M., Lecoanet, D., Perrard, S., Ribeiro, A., Rodet, L., Aurnou, J. M., \& Le Gal, P., 2015. Experimental study of internal wave generation by convection in water, Fluid Dyn. Res., 47(4), 045502.

Le Bars, M., Couston, L. A., Favier, B., Lard, P., Lecoanet, D., \& Le Gal, P., 2020. Fluid dynamics of a mixed convective/stably stratified syste-A review of some recent works, $C$. R. Phys., 21(2), 151-164.

Maas, C., \& Hansen, U., 2019. Dynamics of a terrestrial magma ocean under planetary rotation: A study in spherical geometry, Earth Planet. Sci. Lett., 513, 81-94.

Manglik, A., Wicht, J., \& Christensen, U. R., 2010. A dynamo model with double diffusive convection for Mercury's core, Earth Planet. Sci. Lett., 289(3-4), 619-628.

Melchior, P. J., Crossley, D. J., Dehant, V. P., \& Ducarme, B., 1988. Have inertial waves been identified from the Earth's core?, Structure and Dynamics of Earth's Deep Interior, 46, 1-12. 
Moffatt, H.K., \& Loper, D. E., 1994. The magnetostrophic rise of a buoyant parcel in the Earth's core, Geophys. J Int., 117(2), 394-402.

Monville R., Vidal J., Cébron D., \& Schaeffer, N., 2019. Rotating double-diffusive convection in stably stratified planetary cores, Geophys. J Int., 219(Supplement_1):S195-218.

Mound, J., Davies, C., Rost, S., \& Aurnou, J., 2019. Regional stratification at the top of Earth's core due to core-mantle boundary heat flux variations, Nat. Geosci., 12(7), 575-580.

Mowbray, D. E., \& Rarity, B. S. H., 1967. A theoretical and experimental investigation of the phase configuration of internal waves of small amplitude in a density stratified liquid, J. Fluid Mech., 28(1), 1-16.

Nakagawa, T., 2011. Effect of a stably stratified layer near the outer boundary in numerical simulations of a magnetohydrodynamic dynamo in a rotating spherical shell and its implications for Earth's core, Phys. Earth Planet. Inter., 187(3), 342-352.

Nakagawa, T., 2018. On the thermo-chemical origin of the stratified region at the top of the Earth's core, Phys. Earth Planet. Inter., 276, 172-181.

Ogilvie, G. I., \& Lin, D. N. C., 2004. Tidal dissipation in rotating giant planets, ApJ, 610(1), 477.

Olson, P., 1977. Internal waves in the Earth's core, Geophys. J Int., 51(1), 183-215.

Olson, P., Landeau, M., \& Reynolds, E., 2017. Dynamo tests for stratification below the core-mantle boundary, Phys. Earth Planet. Inter., 271, 1-18.

Olson, P., Landeau, M., \& Reynolds, E., 2018. Outer core stratification from the high latitude structure of the geomagnetic field, Front. Earth Sci., 6, 140.

Ohtani, E., 1985. The primodial terrestrial magma ocean and its implication for stratification of the mantle, Phys. Earth Planet. Inter, 38(1), 70-80.

Roberts, P. H., \& King, E. M., 2013. On the genesis of the Earth's magnetism, Rep. Prog. Phys., 76(9), 096801.

Rogers, T. M., Lin, D. N., \& Lau, H. H. B., 2012. Internal gravity waves modulate the apparent misalignment of exoplanets around hot stars, ApJ, 758(1), L6.

Rogers, T. M., Lin, D. N., McElwaine, J. N., \& Lau, H. H. B., 2013. Internal gravity waves in massive stars: angular momentum transport, $A p J, \mathbf{7 7 2}(1), 21$.

Rosat, S., \& Hinderer, J., 2018. Limits of detection of gravimetric signals on Earth, Sci. Rep., 8(1), 1-8.

Rubie, D. C., Frost, D. J., Mann, U., Asahara, Y., Nimmo, F., Tsuno, K., Kegler, P., Holzheid, A., \& Palme, H., 2011. Heterogeneous accretion, composition and core-mantle differentiation of the Earth, Earth Planet. Sci. Lett., 301, 31-42.

Savaro, C., Campagne, A., Linares, M. C., Augier, P., Sommeria, J., Valran, T., ... \& Mordant, N., 2020. Generation of weakly nonlinear turbulence of internal gravity waves in the Coriolis facility, Phys. Rev. Fluids, 5(7), 073801.

Schmitt, R. W., 1994. Double diffusion in oceanography, Annu. Rev. Fluid Mech., 26(1), 255-285. 
Stanley, S., \& Mohammadi, A., 2008. Effects of an outer thin stably stratified layer on planetary dynamos, Phys. Earth Planet. Inter., 168(3-4), 179-190.

833 Townsend, A. A.,1964. Natural convection in water over an ice surface, Q. J. R. Meteorol. Soc., 90(385),

834 248-259.

835 Vidal, J., \& Schaeffer N., 2015. Quasi-geostrophic modes in the Earth's fluid core with an outer stably stratified layer, Geophys. J Int., 202(3), 2182-2193. 


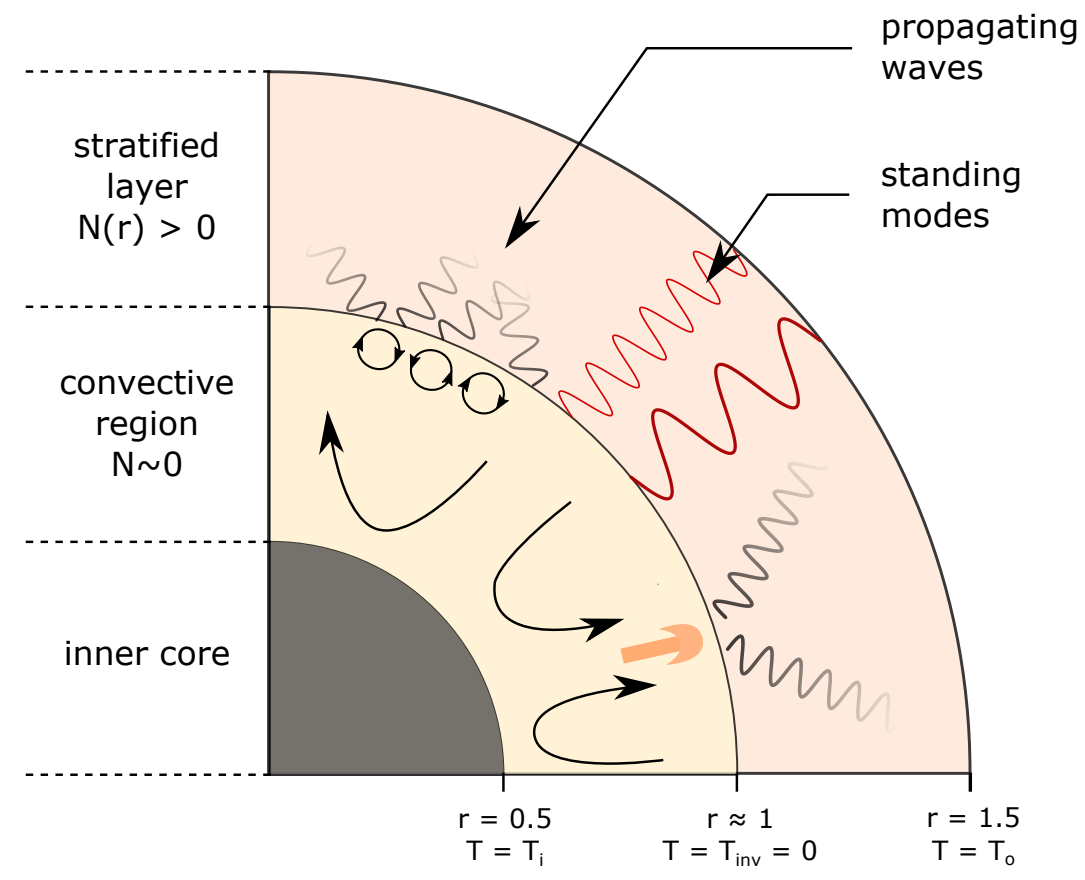

Figure 1. Schematic of the problem studied. A stratified layer occupies the top half of the outer core and lies on top of a vigorously convective region. Reynolds stress in the convective zone excites waves that propagate in the stratified layer. As these waves propagate upwards, they are gradually attenuated. The constructive interactions of propagating waves and their reflections on the top boundary excite and maintain standing modes of various length scales and frequencies, that resonate in the entire cavity defined by the stratified layer. When the damping term in equation (2) is added, the reflection of propagating waves and hence the formation of standing modes is prevented. $N$ denotes the Brunt-Väisälä frequency, as defined in equation (14). Temperatures and radii are indicated in non dimensional units. Although the cartoon is in 2D and shows only a fraction of the sphere, our simulations run in a full 3D spherical shell. 
(a)

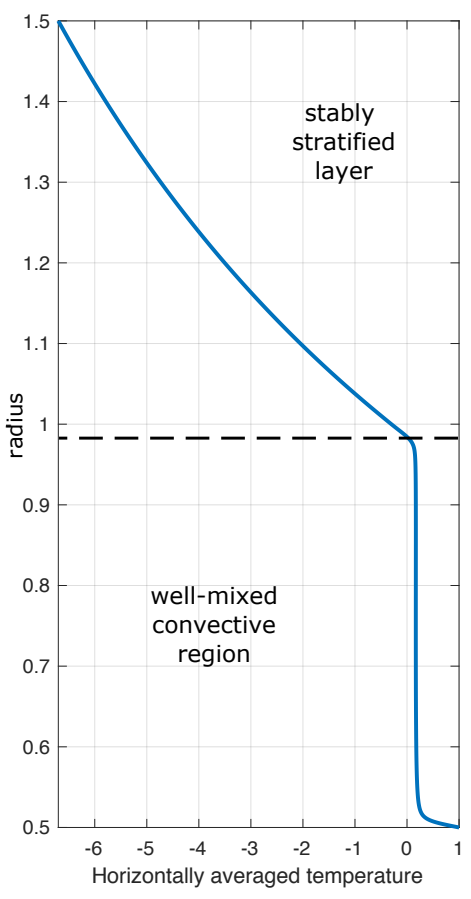

(b)

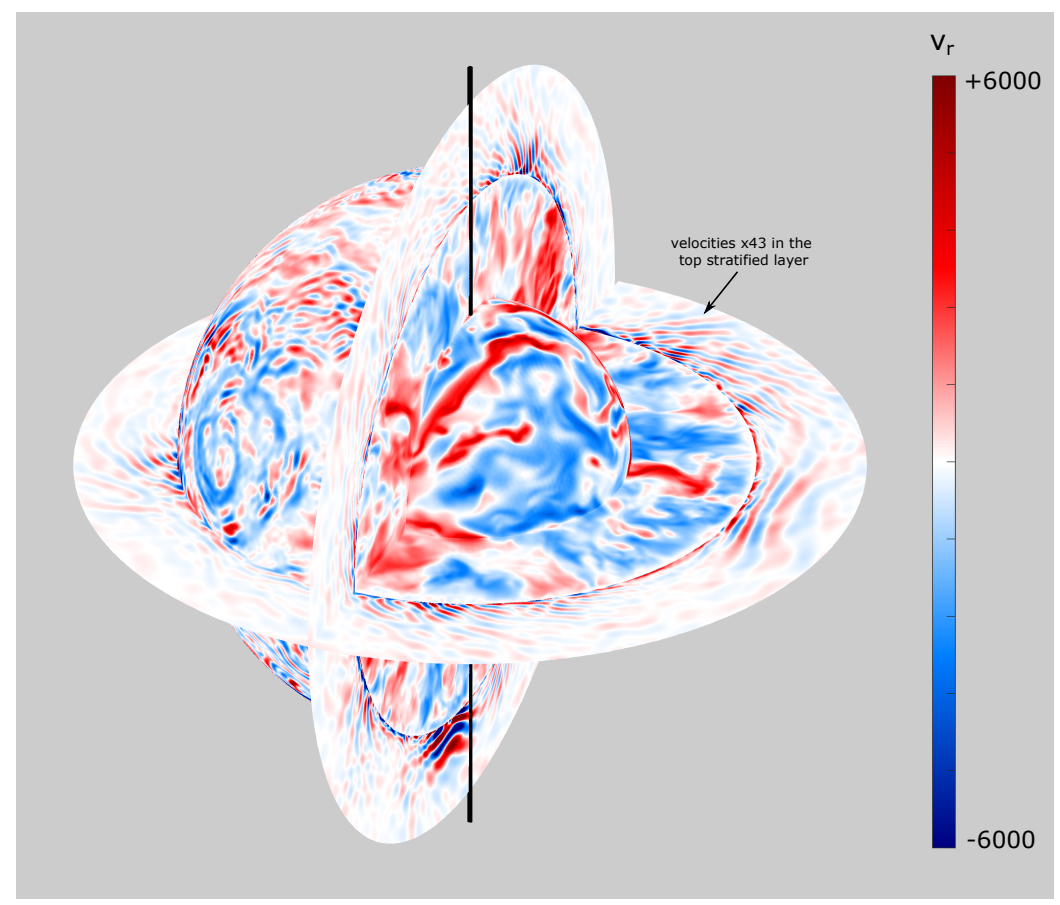

Figure 2. (a): Radial profile of the horizontally averaged temperature for simulation P1. In the well-mixed convective region, at $r \lesssim 1$, the temperature is almost constant while a conductive temperature profile exists in the stratified layer above. The horizontal dashed line corresponds to the radius where $\langle\bar{T}\rangle=0$ and marks the approximate position of the interface. (b): Snapshot showing the radial velocity field for simulation P1. Velocities in the stratified region have been multiplied by a factor 43 , so that they can be visualised together with the velocities in the convective region using the same colorbar. The apparent discontinuity is a result of this color scale adjustment but all numerical variables are continuous. The position of the interface is defined as the radius where the time and angle-averaged temperature $\langle\bar{T}\rangle$ equals the inversion temperature, i.e. $\langle\bar{T}\rangle=0$ in non-dimensional units. See also the corresponding movie attached or at https: //youtu.be/qbPV81I8nkw. 

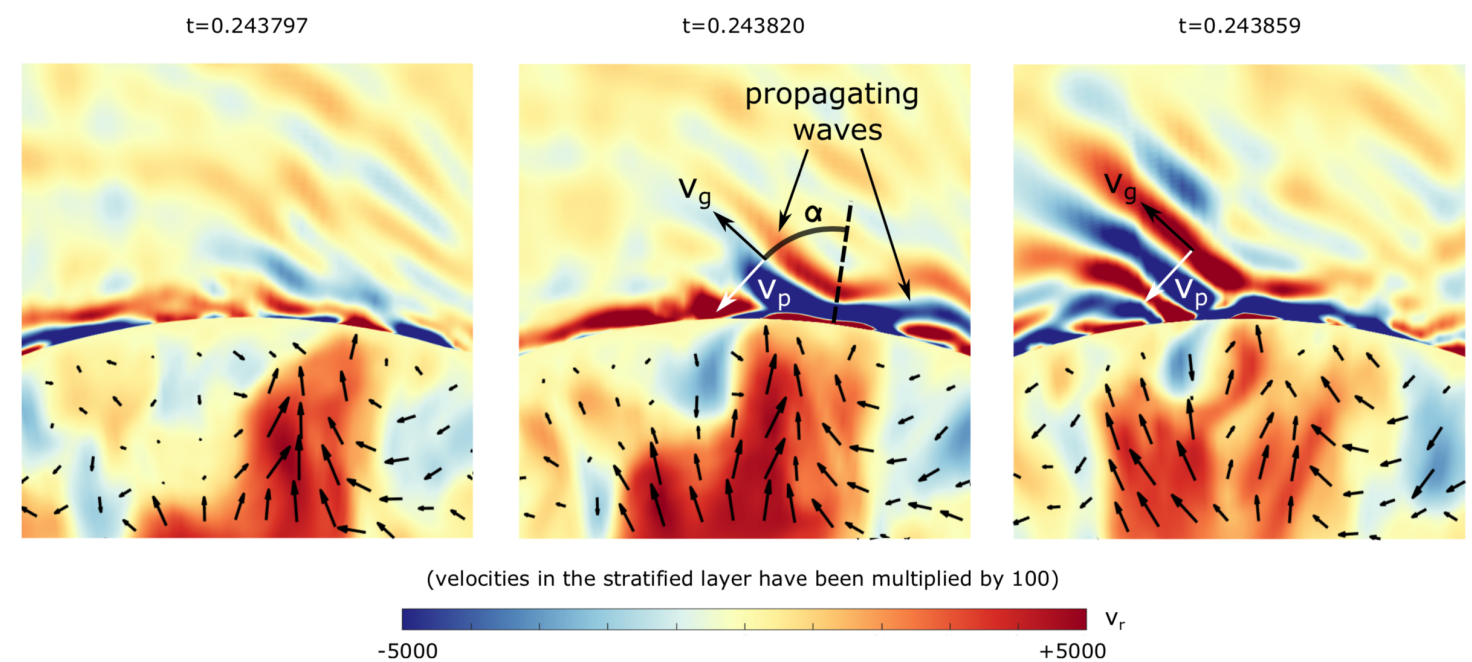

Figure 3. Snapshots of the radial velocity field for simulation P1 at three consecutive times showing a plume impact on the interface with the stratified layer. In a plane containing the impact point, the shape formed by the waves is sometimes referred to as a "half St Andrew's cross" (Mowbray \& Rarity, 1967). $\mathbf{v}_{\mathbf{p}}$ and $\mathbf{v}_{\mathbf{g}}$ denote the phase and group velocities, respectively. Their directions are indicated by arrows. The phase velocity is perpendicular to the group velocity. The energy transport rate and direction is given by the group velocity, at an angle $\alpha$ from the radial direction and oriented away from the convective region. Velocities in the stratified region have been multiplied by a factor 100 for a clearer visualisation. The position of the interface is defined as the radius where the angle-averaged temperature $\langle\bar{T}\rangle(r)$ equals the inversion temperature, i.e. $\langle\bar{T}\rangle=0$ in non-dimensional units. In the convecting region, arrows show the direction and magnitude of the velocity in the plane of the figure.

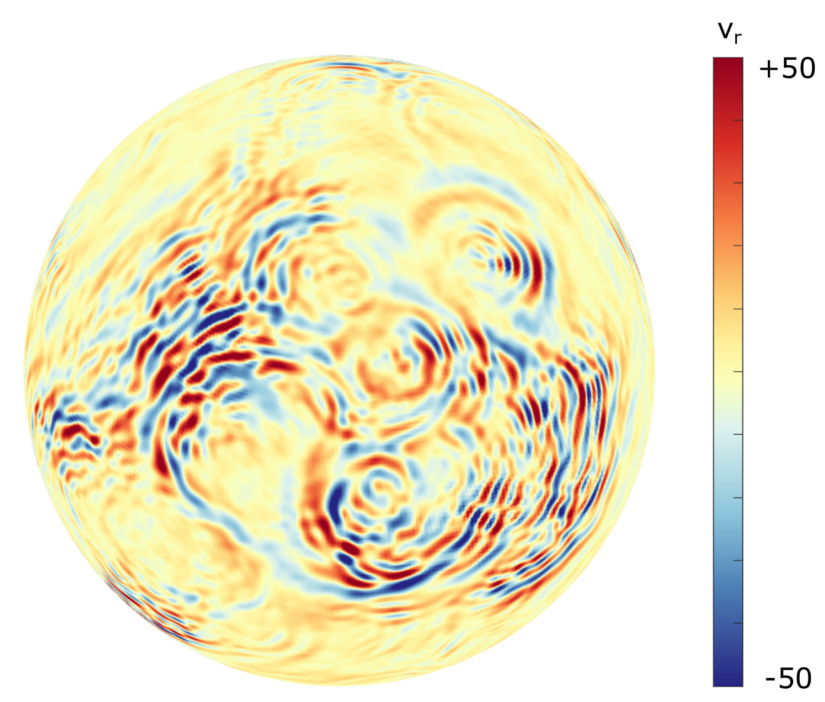

Figure 4. Snapshot of the radial velocity field in the stratified layer at $r=1.066$ for simulation P1. Each concentric structure is the trace of a plume impact that occurred earlier on the interface underneath at $r \approx 1$. 


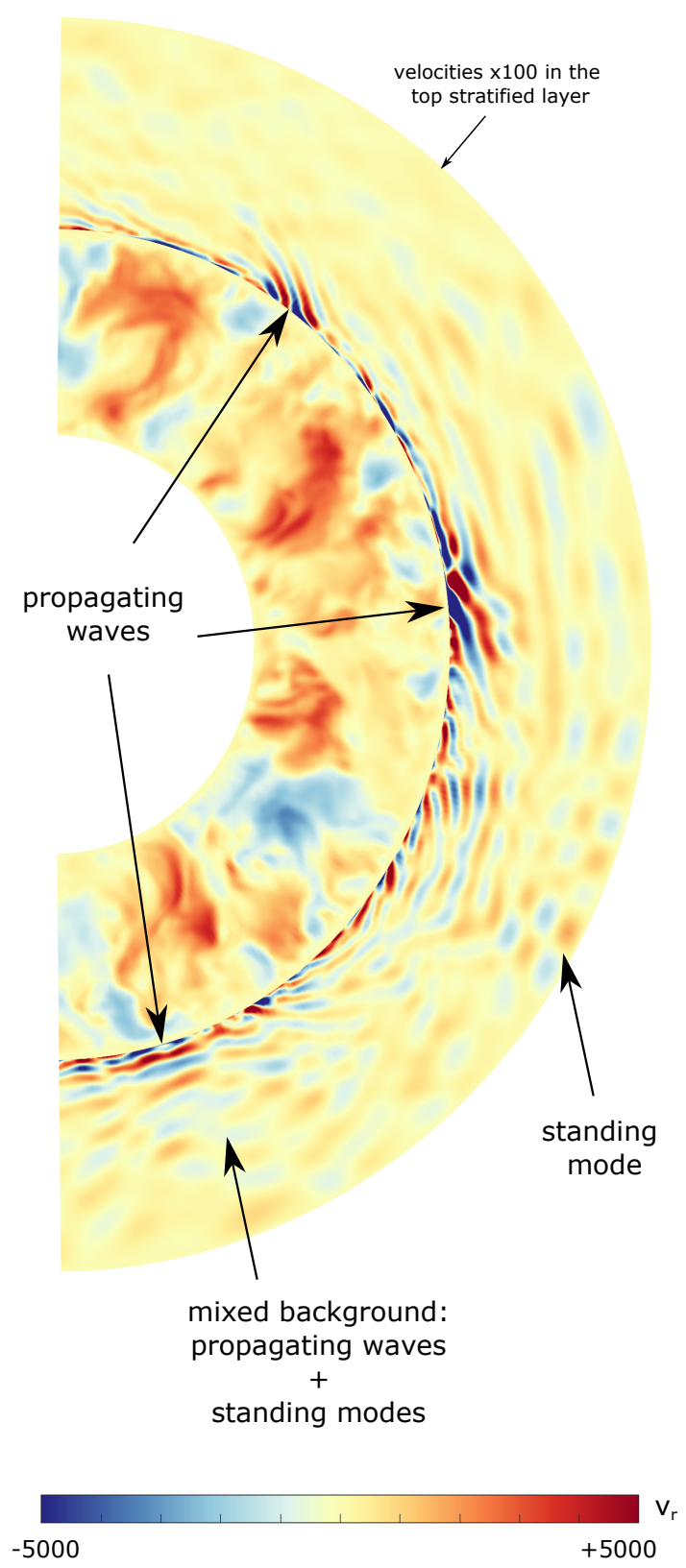

Figure 5. Snapshot of the radial velocity field for simulation P1. Velocities in the stratified region have been multiplied by a factor 100 for a clearer visualisation. The red and blue colors indicate positive and negative signs of the radial velocity, respectively. Propagating waves are excited at the interface with the convective region by Reynolds stresses. These waves propagate in the shell and can reflect on the outer boundary. The interaction of propagating waves with their reflections can lead to multiple standing modes that resonate in the entire cavity formed by the stratified layer. Even though numerous modes are superimposed and can hardly be distinguished individually, one specific mode sometimes dominates locally, revealing itself as a checkerboard pattern like the one pointed by the arrow on the right. 

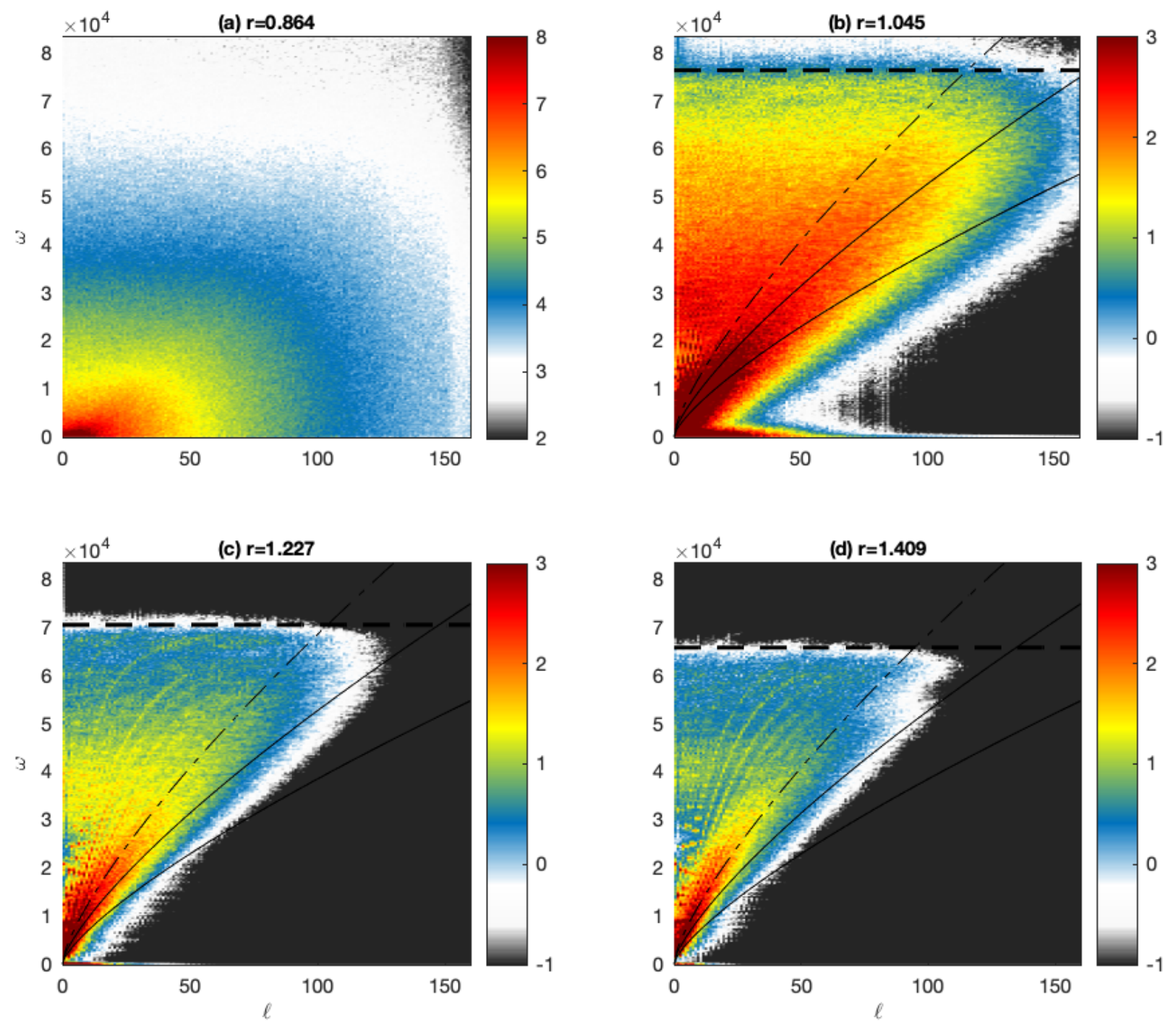

Figure 6. Kinetic energy spectra for simulation P1 in $(\ell, \omega)$ space as defined by equation (24), at different radii, one in the convective region (panel a) and the three others in the stratified layer, with increasing distance from the interface located at $r \approx 1$ (panels $\mathrm{b}$ to $\mathrm{d}$ ). The horizontal dashed lines denote the value of the horizontal mean Brunt-Väisälä frequency at the corresponding radius. The plain lines correspond to iso-values of the attenuation depth $\lambda_{\text {eff }}$ (see equation (31) $), \lambda_{\text {eff }}=0.02$ and $\lambda_{\text {eff }}=0.07$. The dashed-dotted line corresponds to the $\lambda_{\text {eff }}=0.2$ line and approximately separates propagating waves (below the line) and modes (above the line). The colorbars are in logarithmic scale and span several orders of magnitude. The colorscale has been cut at a lower bound to focus on the most energetic signals. The three colorbars in panels (b), (c) and (d) are identical while panel (a) focuses on much larger amplitudes, characteristic of the convective region. 


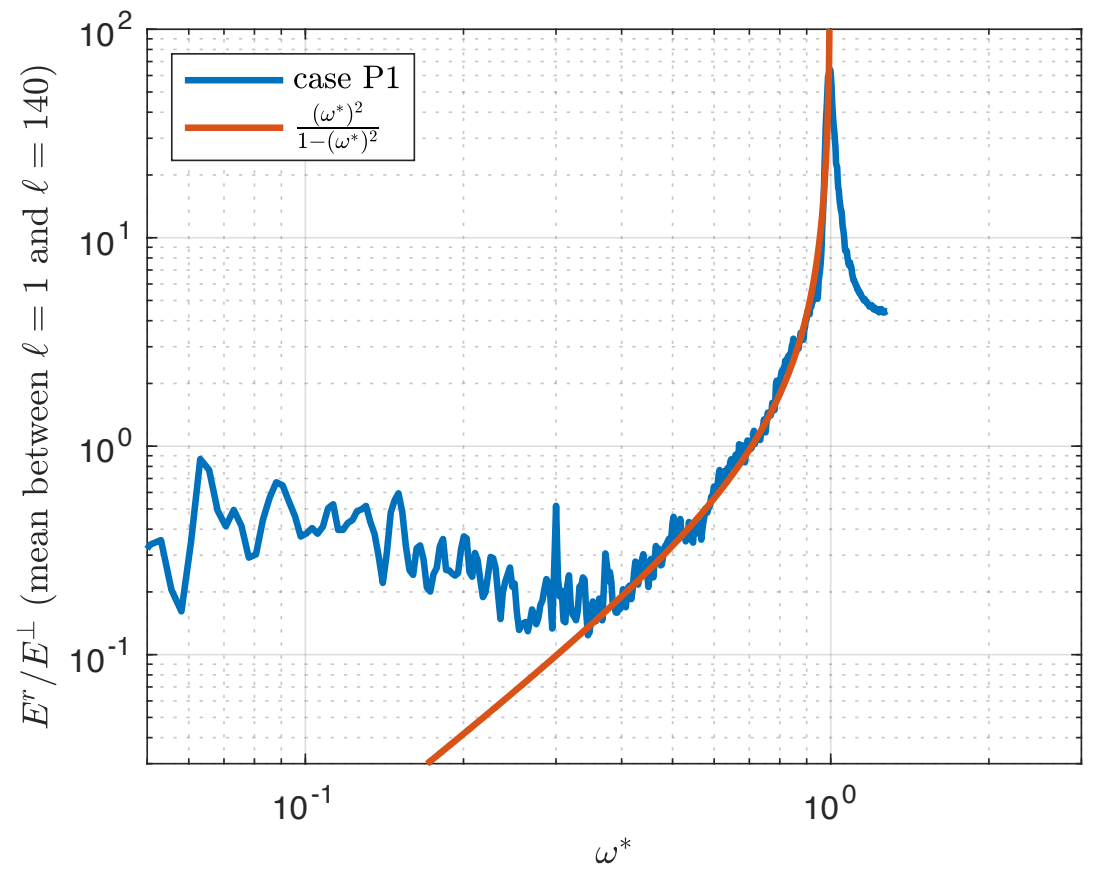

Figure 7. Ratio of the frequency spectra $E^{r} / E^{\perp}$, at $r=1.318$, averaged between $\ell=1$ and $\ell=140$. $\omega^{*}=\omega / N$ is the frequency normalized by the Brunt-Väisälä frequency. The red curve shows the law 25] (see main text), which is valid only in the GW region. The curve matches the spectrum in the waves region, close to $\omega=N$ and below. The mismatch at lower frequencies indicates that the low-frequency part does not contain only linear GW. 

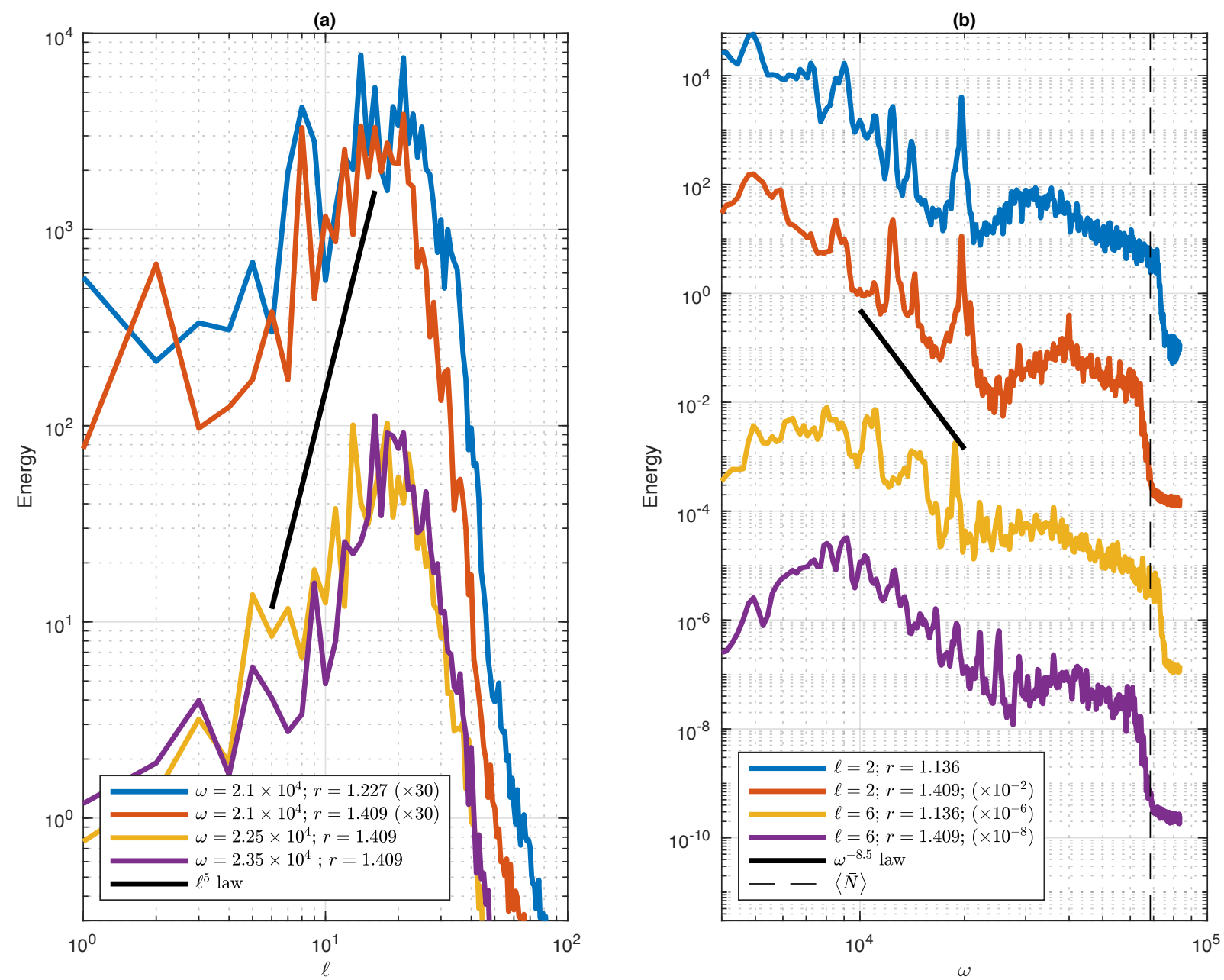

Figure 8. (a): $\ell$ spectra at two radii and for two fixed frequencies. (b): $\omega$ spectra for two radii and two fixed values of $\ell$. The vertical dashed line corresponds to $\omega=\bar{N}$. Two of the spectra in (a) and three of the spectra in (b) have been shifted upwards/downwards to better visualize the modes (the spikes that superimpose to the global drop in kinetic energy) and to compare slopes of the different spectra. The black lines indicate the slopes of the power laws predicted by the theory of Lecoanet \& Quataert (2013). 

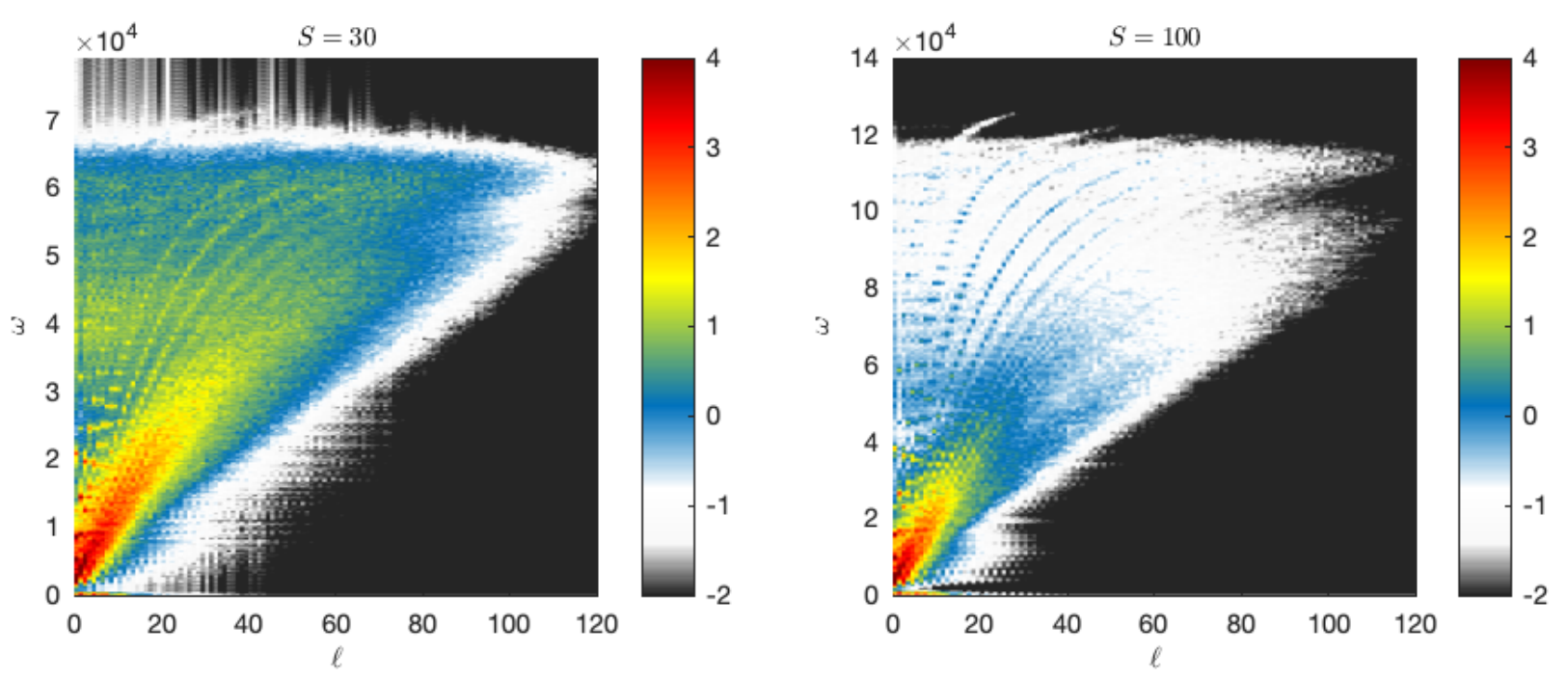

Figure 9. $(\ell, \omega)$ spectra at $r=1.136$ for simulation $\mathrm{P} 1$ ( $S=30$, left panel) and simulation P1_S100 $(S=100$, right panel). Note that the colorbars are the same for both subfigures and span several orders of magnitude.
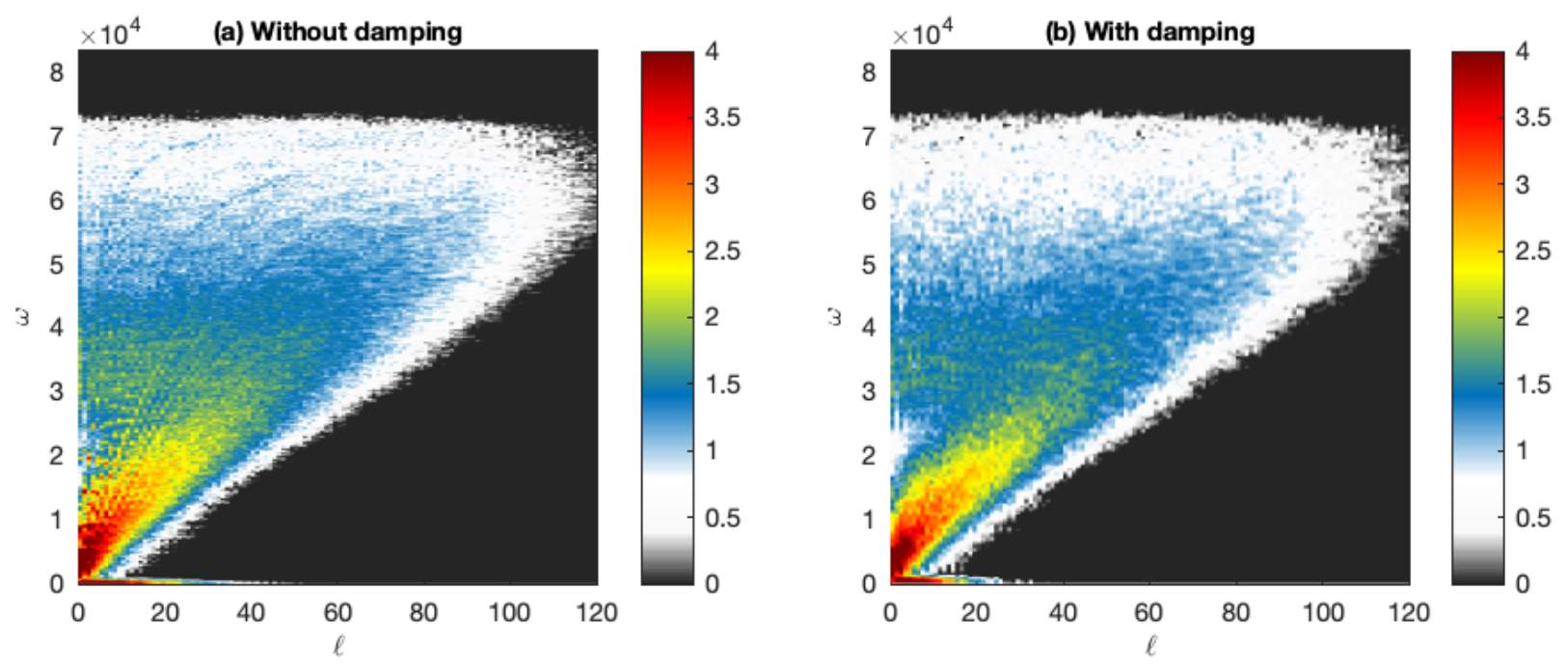

Figure 10. Kinetic energy spectra in $(\ell, \omega)$ space as defined by equation 24) at $r=1.136$. a: simulation P1 (without damping term). b: simulation P1d (with damping). Note that the colorbars span several orders of magnitude and are in logarithmic scale. 

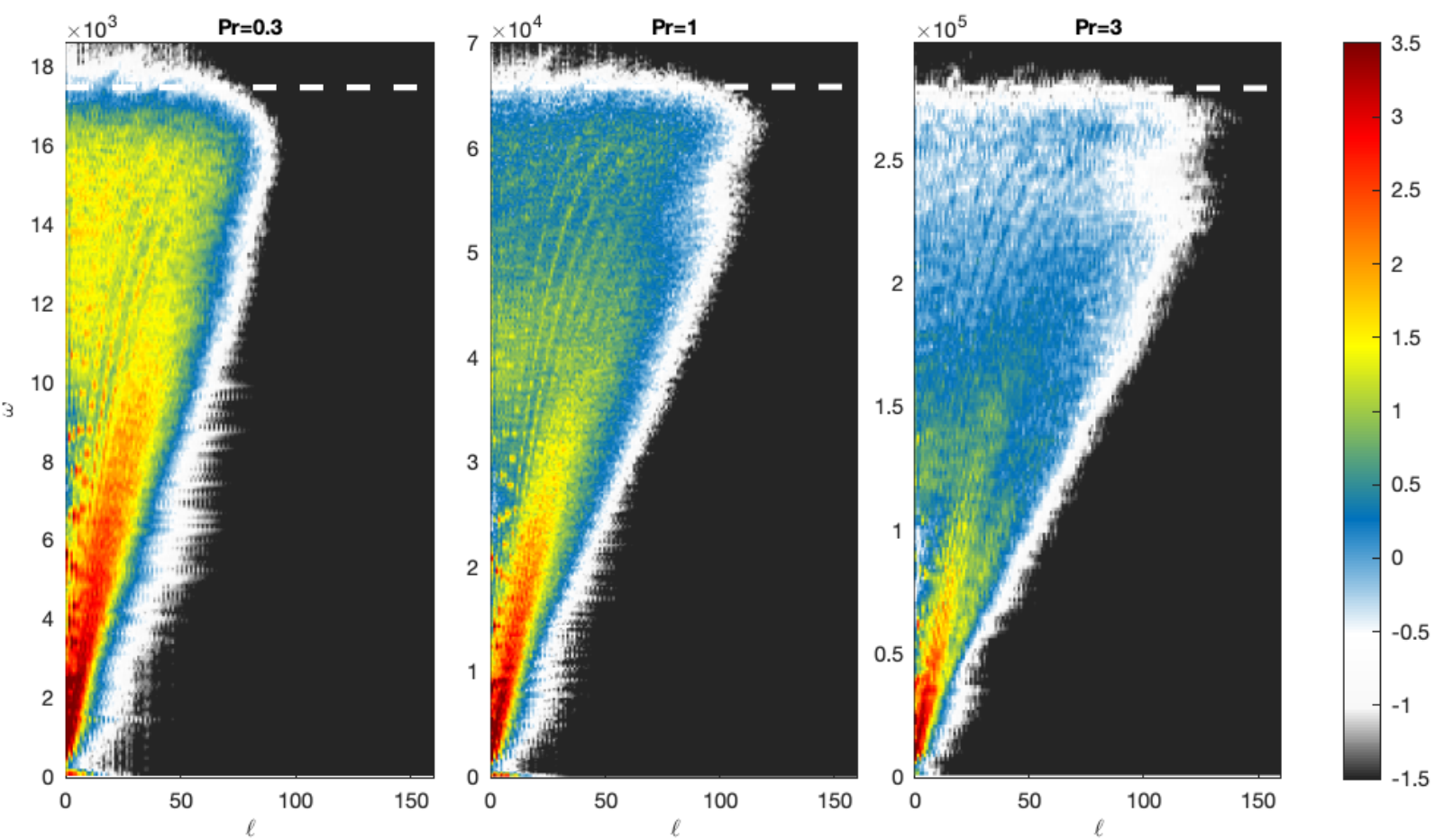

Figure 11. $(\ell, \omega)$ spectra at $r=1.318$ for simulations $\mathrm{P} 03(\operatorname{Pr}=0.3), \mathrm{P} 1(\operatorname{Pr}=1)$ and $\mathrm{P} 3(\operatorname{Pr}=3)$. The horizontal dashed lines indicate the Brunt-Väisälä frequency at the radius considered. 

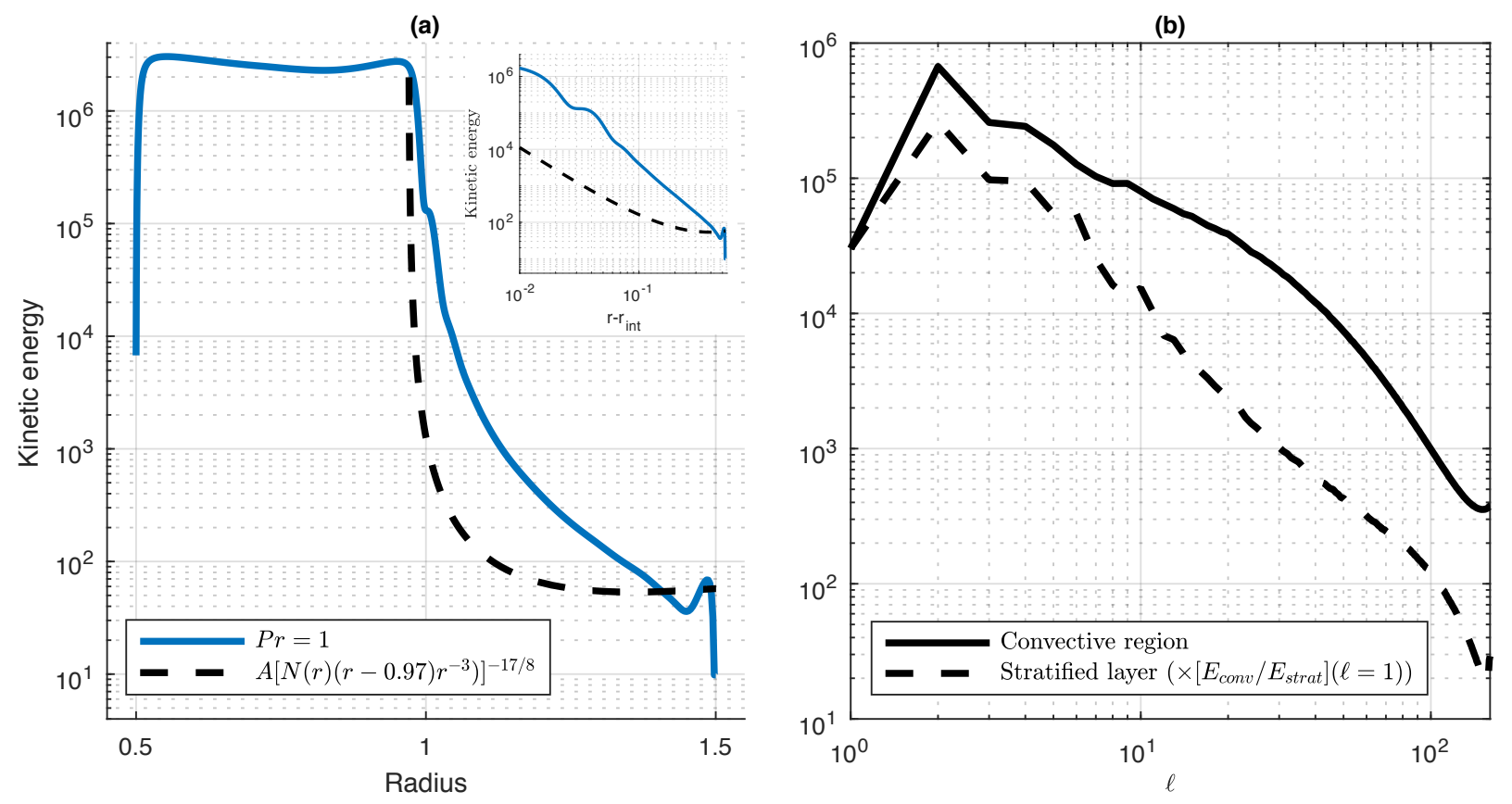

Figure 12. (a): radial profile of the kinetic energy. The dashed line shows the law in equation 39] with a prefactor $A=8 \times 10^{10}$. The inserted subfigure shows the behavior with the distance to the interface radius $\left(r_{\text {int }}=0.97\right)$ in log-log scale.(b): $\ell$-spectra of the kinetic energy in the convective region $\left(r \leq r_{\text {int }}-0.025\right)$ and in the stratified layer $\left(r \geq r_{\text {int }}+0.025\right)$, for simulation P1. To allow for a better comparison, the spectrum in the stratified layer has been renormalized by a factor $E_{\text {conv }}(\ell=1) / E(\ell=1)=89.3$. 

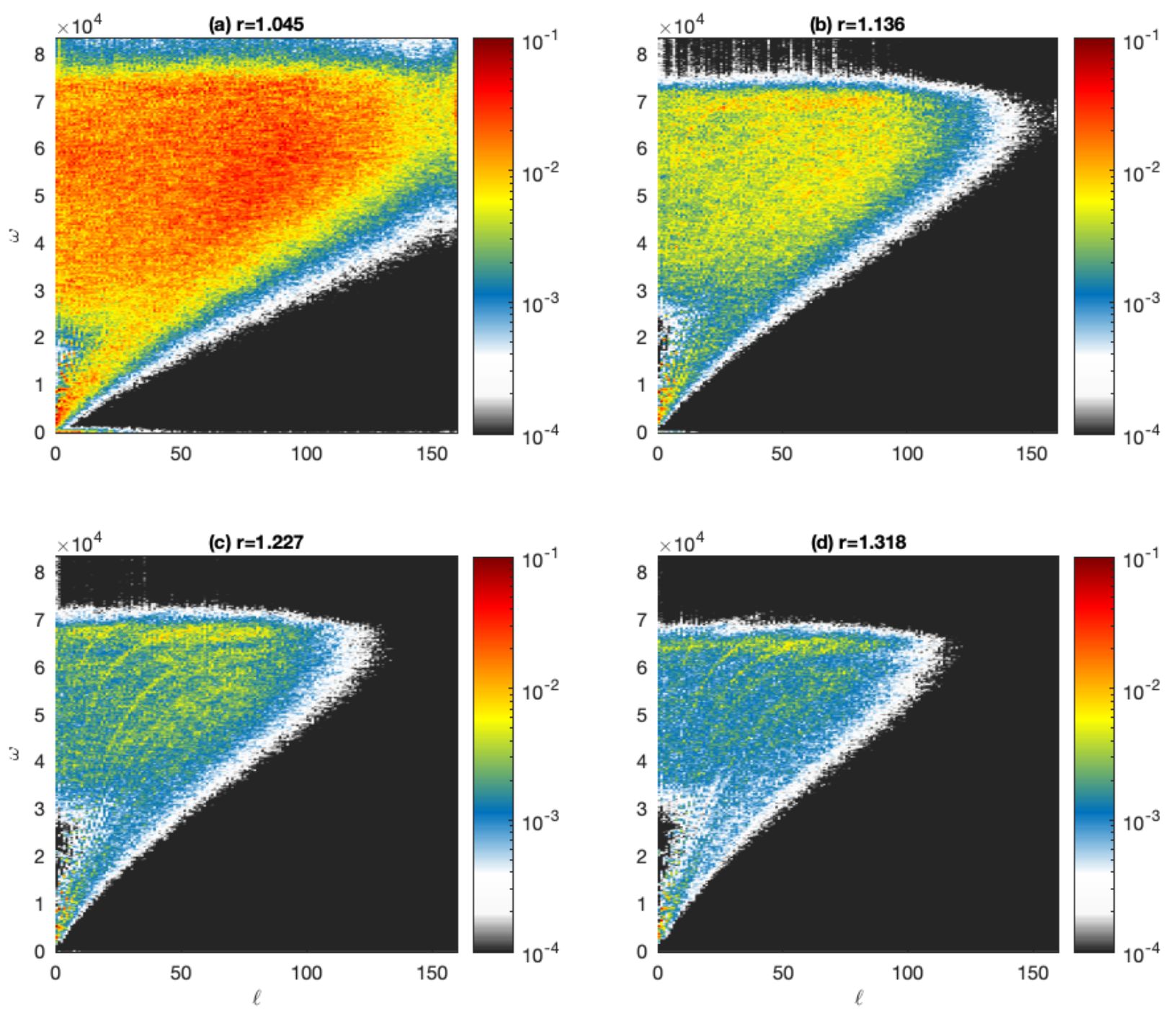

Figure 13. Kinetic energy transmission factor between the convective region at $r=0.863$ and the stratified layer at different radii. 


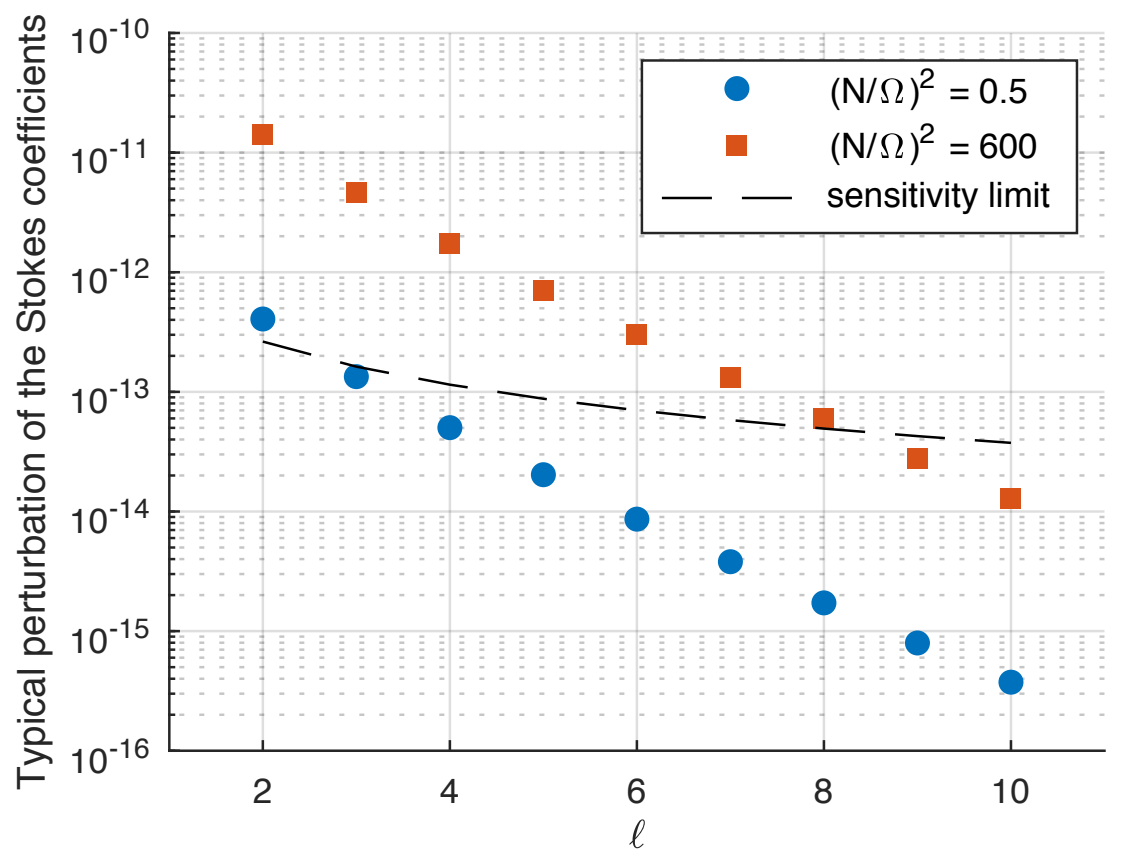

Figure 14. Order of magnitude of the change in the Stokes coefficients for the first spherical harmonics degrees and for two different values of $N$ spanning the range proposed in the literature. The dashed line indicates approximately the sensitivity limit of present-day gravimeters, about 1 nGal (Rosat \& Hinderer, 2018). 